\title{
Testing epidemic change in nearly nonstationary process with statistics based on residuals
}

\author{
J. Markevičiūtè $\dot{e}^{* 1}$ A. Račkauskas ${ }^{\dagger 1}$, and Ch. Suquet ${ }^{\ddagger 2}$ \\ ${ }^{1}$ Faculty of Mathematics and Informatics, Vilnius University, Naugarduko str. \\ 24, LT-03225 Vilnius, Lithuania \\ ${ }^{2}$ Laboratoire P. Painlevé, UMR 8524 CNRS Université Lille I, Bât. M2, Cité \\ Scientifique, F-59655 Villeneuve d'Ascq Cedex, France
}

Dated: January 17, 2021

\begin{abstract}
We study an epidemic type change in innovations of a first order autoregressive process $y_{n, k}=\phi_{n} y_{n, k-1}+\varepsilon_{k}+a_{n, k}$, where $\phi_{n}$ is either a constant in $(-1,1)$ or a sequence in $(0,1)$, converging to 1 . For $k$ inside some unknown interval $\mathbb{I}_{n}^{*}=\left(k^{*}, k^{*}+\ell^{*}\right], a_{n, k}=a_{n}$ while $a_{n, k}=0$ for $k$ outside $\mathbb{I}_{n}^{*}$. When $a_{n} \neq 0$, we have an epidemic deviation from the usual (zero) mean of innovations. Since innovations are not observed, we build uniform increments statistics on residuals $\left(\widehat{\varepsilon}_{k}\right)$ of the process $y_{n, k}$. We assume that innovations $\left(\varepsilon_{k}\right)$ are regularly varying with index $p \geq 2$ or satisfies integrability condition $\lim _{t \rightarrow \infty} t^{p} \mathbb{P}\left(\left|\varepsilon_{1}\right|>t\right)=0$ for $p>2$ and $\mathbb{E} \varepsilon_{k}^{2}<\infty$ for $p=2$. We find the limit distributions of the tests under no change and prove consistency under short epidemics that is $\ell^{*}=O\left(n^{\beta}\right)$ for some $0<\beta \leq 1 / 2$.
\end{abstract}

Keywords: autoregressive process; epidemic change; regular variation; Brownian motion; uniform increments statistics.

$M S C: 62 \mathrm{M} 10 ; 62 \mathrm{~F} 03$.

\footnotetext{
*Electronic address: jurgita.markeviciute@mif .vu.1t; Corresponding author

$\dagger$ Electronic address: alfredas.rackauskas@mif.vu.lt

${ }^{\ddagger}$ Electronic address: Charles.Suquet@math.univ-lille1.fr
} 


\section{Introduction}

Suppose we are given a sequence of observations $\left(\varepsilon_{k}\right)$ that are assumed to be independent identically distributed (i.i.d.) random variables with zero mean, except, maybe, for a short interval, where the mean of the corresponding observations is a nonzero constant. Such model can be interpreted as an epidemic one. The nonzero mean corresponds to an epidemic deviation from the usual state. The length of the interval describes the duration of the epidemic. The question is how to decide whether such an interval is present. To the best of our knowledge, this kind of problem was formulated for the first time by Levin and Kline [1] in the context of abortion epidemiology. Simultaneously, epidemic type models were introduced by Commenges, Seal and Pinatel [2] in connection with experimental neurophysiology. Models with an epidemic type change in the mean were also used for detecting changed segments in non-coding DNA sequences [3] and for studying structural breaks in econometric contexts [4]. Levin and Kline [1] proposed the test statistic $\max _{1 \leq \ell \leq n} \max _{0 \leq k \leq n-\ell}\left(\sum_{j=k+1}^{k+\ell} \varepsilon_{j}-\ell \delta / 2\right)$, where $\delta>0$ represents the smallest increment in the mean which is sufficiently important to be detected. Another type of statistics can be constructed by normalizing the sums $\sum_{j=k+1}^{k+\ell} \varepsilon_{j}$ according to our guess about the length of the epidemic state. In this way we arrive at the following multiscale type statistic

$$
T_{n}\left(\varepsilon_{1}, \ldots, \varepsilon_{n}\right)=\max _{1 \leq \ell \leq n} \ell^{-\alpha} \max _{1 \leq k \leq n-\ell}\left(\sum_{j=k+1}^{k+\ell} \varepsilon_{j}\right),
$$

where $0 \leq \alpha \leq 1$. Large values of this statistic indicate the presence of an epidemic state. We refer to [5] for various asymptotic results for this type of statistics. Yet another type of statistic based on ranks and signs of observations where suggested by Gombay [6]. She also pointed out that despite the fact that the epidemic model can be formulated as multiple change-point model, tests constructed with account of a particular form of changes may have bigger power.

The problem that we are concerned with in this paper is addressed to a situation where the sequence $\left(\varepsilon_{k}\right)$ appears as innovations in a certain time series model. So that we cannot observe $\left(\varepsilon_{k}\right)$ directly. What usually we have at hands are residuals $\left(\widehat{\varepsilon}_{k}\right)$ obtained by an estimation procedure of the model under consideration. This suggests that testing for an epidemic state in the sequence $\left(\varepsilon_{k}\right)$ can be based on residuals $\left(\widehat{\varepsilon}_{k}\right)$. To be more precise, assume we are given a sample $y_{n, 1}, \ldots, y_{n, n}$ for a fixed $n$, generated from the first order autoregressive process

$$
y_{n, k}=\phi_{n} y_{n, k-1}+\varepsilon_{k}+a_{n, k}, \quad k=1, \ldots, n, \quad n \geq 1, \quad y_{n, 0}=0,
$$


where the unknown coefficient $\phi_{n}$ is either a constant $\phi$ in $(-1,1)$ or a sequence of constants $\phi_{n} \in(0,1)$ and $\phi_{n}$ tends to 1 , as $n \rightarrow \infty$. The innovations $\left(\varepsilon_{k}, k \leq n\right)$ are unobservable, centered, at least square integrable random variables. In what follows we denote

$$
\gamma_{n}:=n\left(1-\phi_{n}\right)
$$

and assume throughout the paper that $\lim _{n \rightarrow \infty} \gamma_{n}=\infty$. When all the $a_{n, k}$ are null and $\phi_{n}$ tends to 1 , the process $y_{n, k}$ is called nearly nonstationary. We refer to Giraitis and Philips [7] for a study of the asymptotic behaviour of such a process.

The aim of this paper is to propose tests for the null hypothesis

$$
H_{0}: \quad a_{n, 1}=\cdots=a_{n, n}=0
$$

against the epidemic alternative:

$$
\begin{aligned}
H_{A}: & \text { there exist } 0 \leq k_{n}^{*}<n, \quad 1 \leq m_{n}^{*} \leq n \text { such that } \\
& a_{n, k}=a_{n} \neq 0 \text { for } k \in \mathbb{I}_{n}^{*} \text { whereas } a_{n, k}=0 \text { for } k \notin \mathbb{I}_{n}^{*},
\end{aligned}
$$

where $\mathbb{I}_{n}^{*}=\left\{k_{n}^{*}+1, \ldots, m_{n}^{*}\right\}$. The value $a_{n}$ during the period $\mathbb{I}_{n}^{*}$ is interpreted as an epidemic deviation from the usual (zero) mean of innovations and $\ell_{n}^{*}=m_{n}^{*}-k_{n}^{*}$ is the duration of the epidemic state.

Set for $\alpha \in[0,1)$ and any real numbers $x_{1}, \ldots, x_{n}$ :

$$
T_{\alpha, n}\left(x_{1}, \ldots, x_{n}\right)=\max _{1 \leq \ell \leq n} \ell^{-\alpha} \max _{1 \leq k \leq n-\ell}\left|\sum_{j=k+1}^{k+\ell} x_{j}-\frac{\ell}{n} \sum_{j=1}^{n} x_{j}\right| .
$$

Denote

$$
T_{\alpha, n}=T_{\alpha, n}\left(\varepsilon_{1}, \ldots, \varepsilon_{n}\right),
$$

and

$$
\widehat{T}_{\alpha, n}=T_{\alpha, n}\left(\widehat{\varepsilon}_{1}, \ldots, \widehat{\varepsilon}_{n}\right),
$$

where $\left(\widehat{\varepsilon}_{k}\right)$ are residuals of the model (1) defined by

$$
\widehat{\varepsilon}_{k}=y_{n, k}-\widehat{\phi}_{n} y_{n, k-1}, \quad k=1, \ldots, n,
$$

and $\widehat{\phi}_{n}$ is the least square estimator of $\phi_{n}$ :

$$
\widehat{\phi}_{n}=\frac{\sum_{k=1}^{n} y_{n, k} y_{n, k-1}}{\sum_{k=1}^{n} y_{n, k-1}^{2}} .
$$

Roughly speaking, under $H_{A}$, the probability of detection of the epidemic is an increasing function of the amplitude of the jump $\left|a_{n}\right|$ and of the length 
of the epidemic interval $\ell^{*}$. If we take $a_{n}=a$ constant and assume that $\ell^{*}=\theta n^{\beta}$, for some $0<\beta \leq 1$, then the choice $\alpha=0$ in the definition of $T_{\alpha, n}$ leads to the classical CUSUM procedure which allows to detect epidemic of length $\ell^{*}=\theta n^{\beta}$ when $\beta>1 / 2$. The asymptotic behavior of $T_{0, n}$ and $\widehat{T}_{0, n}$ are deduced from a functional central limit theorem in the space $\mathrm{C}[0,1]$. The main interest of the statistics $\widehat{T}_{\alpha, n}$ is that the possibility to choose $\alpha>0$, subject to some additional integrability condition on the innovations, allows the detection of shorter epidemics, of length $\ell^{*}=\theta n^{\beta}$ with $\beta<1 / 2$. For a study of the epidemic detection in an i.i.d. sample via Hölderian techniques, we refer to [5]. $\left(\varepsilon_{k}\right)$.

We investigate the limit behavior of $\widehat{T}_{\alpha, n}$ for two classes of innovations

Definition 1.1. Let $p>0$. We say that a random variable $X$ belongs to the class

- $\mathcal{L}_{p, \infty}$, if $\sup _{t>0} t^{p} \mathbb{P}(|X|>t)<\infty$,

- $\mathcal{L}_{p, \infty}^{o}$, if $\lim _{t \rightarrow \infty} t^{p} \mathbb{P}(|X|>t)=0$,

- $\mathcal{L}_{p}$, if $\mathbb{E}|X|^{p}<\infty$.

It is well known that for $0<r<p, \mathcal{L}_{p} \subset \mathcal{L}_{p, \infty}^{o} \subset \mathcal{L}_{p, \infty} \subset \mathcal{L}_{r}$.

Definition 1.2. The random variable $X$ is regularly varying with index $p>0$ (denoted $X \in \mathrm{RV}_{p}$ ) if there exists a slowly varying function $L$ such that the distribution function $F(t)=P(X \leq t)$ satisfies the tail balance condition

$$
F(-x) \sim b L(x) x^{-p} \text { and } \quad 1-F(x) \sim a L(x) x^{-p}, \quad \text { as } \quad x \rightarrow \infty,
$$

where $a, b \in(0,1)$ and $a+b=1$.

We refer to [8] for an encyclopedic treatment of regular variation. We note that if $0<r<p$, then $\mathrm{RV}_{p} \subset \mathcal{L}_{r, \infty}^{o}$. Moreover, if $L(x) \rightarrow 0$ as $x \rightarrow \infty$, then $\operatorname{RV}_{p} \subset \mathcal{L}_{p, \infty}^{o}$. Further, if $\varepsilon_{1} \in \mathrm{RV}_{p}$ then define

$$
b_{n}=\inf \left\{x>0: \mathbb{P}\left(\left|\varepsilon_{1}\right| \leq x\right) \geq 1-1 / n\right\} .
$$

It easily follows from tail condition that there is a slowly varying function $v(n), n \in \mathbb{N}$, such that

$$
b_{n} \sim n^{1 / p} v(n) \text { as } n \rightarrow \infty .
$$


Throughout the paper, $\underset{n \rightarrow \infty}{\stackrel{\mathcal{D}}{\longrightarrow}}$ means convergence in distribution.

The paper is organized as follows. In Section 2 we establish limits of distributions of the test statistics under null hypothesis. Section 3 contains consistency analysis of the test statistics. All the proofs of technical intermediate results are detailed in the appendix.

\section{Limit behavior of test statistics under null hypothesis}

From now on, for any $p \geq 2$, we set

$$
\alpha_{p}:=\frac{1}{2}-\frac{1}{p} .
$$

Since we assume $H_{0}$ true throughout this section, the data generating process $y_{n, k}$ is given by

$$
y_{n, k}=\phi_{n} y_{n, k-1}+\varepsilon_{k}, \quad k=1, \ldots, n, \quad n \geq 1, \quad y_{n, 0}=0 .
$$

The following lemma is the key to connect the asymptotic behavior of $\widehat{T}_{\alpha, n}$ to the one of $T_{\alpha, n}$. Its proof being quite long and technical is deferred to the appendix (section $\mathrm{A})$.

Lemma 2.1. Assume that the $\varepsilon_{i}$ 's are i.i.d. random variables and the $y_{n, k}$ 's satisfy (9).

a) If $\varepsilon_{1} \in \mathrm{RV}_{p}$ for some $p \geq 2$ and

$$
\lim _{n \rightarrow \infty} n\left(1-\phi_{n}\right)=\infty .
$$

then for any $\alpha \in\left(\alpha_{p}, 1\right]$ with $\alpha_{p}$ defined by (8),

$$
\begin{aligned}
\left(1-\phi_{n}\right) T_{\alpha, n}\left(y_{n, 0}, \ldots, y_{n, n-1}\right) & =T_{\alpha, n}\left(\varepsilon_{1}, \ldots, \varepsilon_{n}\right)+O_{P}\left(b_{n}\right) \\
T_{\alpha, n}\left(\widehat{\varepsilon}_{1}, \ldots, \widehat{\varepsilon}_{n}\right) & =T_{\alpha, n}\left(\varepsilon_{1}, \ldots, \varepsilon_{n}\right)+o_{P}\left(b_{n}\right) .
\end{aligned}
$$

where $b_{n}$ is defined by (6).

b) If $\varepsilon_{1} \in \mathcal{L}_{2}$ (then $p=2$ ) or $\varepsilon_{1} \in \mathcal{L}_{p, \infty}^{o}$ for some $p>2$, and

$$
\liminf _{n \rightarrow \infty} n^{1-\delta}\left(1-\phi_{n}\right)>0 \quad \text { for some } \delta>0 \text {, }
$$

then for any $\alpha \in\left[0, \alpha_{p}\right]$,

$$
\begin{aligned}
\left(1-\phi_{n}\right) T_{\alpha, n}\left(y_{n, 0}, \ldots, y_{n, n-1}\right) & =T_{\alpha, n}\left(\varepsilon_{1}, \ldots, \varepsilon_{n}\right)+O_{P}\left(n^{1 / 2-\alpha}\right) \\
T_{\alpha, n}\left(\widehat{\varepsilon}_{1}, \ldots, \widehat{\varepsilon}_{n}\right) & =T_{\alpha, n}\left(\varepsilon_{1}, \ldots, \varepsilon_{n}\right)+o_{P}\left(n^{1 / 2-\alpha}\right) .
\end{aligned}
$$


Theorem 2.2. Assume that the $\varepsilon_{i}$ 's are mean zero i.i.d. random variables in $\mathcal{L}_{2}$ (then $p=2$ ) or in $\mathcal{L}_{p, \infty}^{o}$ for some $p>2$, and that the $y_{n, k}$ 's satisfy (9) with $\phi_{n}$ satisfying (13). Then for any $\alpha \in\left[0, \alpha_{p}\right]$,

$$
n^{-1 / 2+\alpha} \sigma^{-1} \widehat{T}_{\alpha, n} \underset{n \rightarrow \infty}{\stackrel{\mathcal{D}}{\longrightarrow}} T_{\alpha, \infty}(W):=\max _{0<h<1} h^{-\alpha} \max _{0 \leq t \leq 1-h}\left|W_{t+h}-W_{t}-h W_{1}\right|,
$$

where $\sigma^{2}=\mathbb{E} \varepsilon_{1}^{2}$ and $W=\left\{W_{t}, 0 \leq t \leq 1\right\}$ is a standard Brownian motion.

Proof. Let $\mathrm{H}_{\alpha}^{o}$ be the set of continuous functions $f:[0,1] \rightarrow \mathbb{R}$ such that $\omega_{\alpha}(f, \delta):=\sup \left\{|f(t)-f(s)||t-s|^{-\alpha}: 0<|t-s| \leq \delta\right\}$ tends to zero as $\delta$ tends to zero. For $0 \leq \alpha<1, \mathrm{H}_{\alpha}^{o}$ is equipped with the $\alpha$-Hölder norm $\|f\|_{\alpha}:=|f(0)|+\omega_{\alpha}(f, 1)$. It is known that if $f$ is a polygonal line, then the supremum in the definition of $\omega_{\alpha}(f, 1)$ is reached at two vertices, see e.g. [9, Lem. A.2]. Hence $n^{\alpha} T_{\alpha, n}\left(\varepsilon_{1}, \ldots, \varepsilon_{n}\right)$ is exactly the $\alpha$-Hölder norm of the random polygonal function $W_{n}-W_{n}(1) \mathrm{Id}$, where Id is the identity function on $[0,1]$ and

$$
W_{n}(t)=\sum_{k=1}^{[n t]} \varepsilon_{k}+\{n t\} \varepsilon_{[n t]+1}, \quad t \in[0,1]
$$

where $\{n t\}$ is the fractional part of $n t$. Since $\varepsilon_{1} \in \mathcal{L}_{p, \infty}^{o}, n^{-1 / 2} \sigma^{-1} W_{n}$ converges in distribution to the standard Brownian motion $W$ in $\mathrm{H}_{\alpha_{p}}^{o}$, where $\alpha_{p}=1 / 2-1 / p$, see [10]. By topological inclusions of Hölder spaces, the same convergence holds in any Hölder space of exponent $0<\alpha<\alpha_{p}$ if $\alpha_{p}>0$. In the special case $\alpha=0, \mathrm{H}_{\alpha}^{o}$ is isomorphic to $\mathrm{C}[0,1]$ and the convergence of $n^{-1 / 2} \sigma^{-1} W_{n}$ is simply the classical invariance principle from DonskerProkhorov. Since the linear operator $B: f \mapsto f-f(1) \mathrm{Id}$ is continuous on $\mathrm{H}_{\alpha}^{o}$, $B\left(n^{-1 / 2} \sigma^{-1} W_{n}\right)$ converges in distribution on $\mathrm{H}_{\alpha}^{o}$ to $B(W)$, for $0 \leq \alpha \leq \alpha_{p}$. Hence by continuous maping, $n^{-1 / 2+\alpha} T_{\alpha, n}\left(\varepsilon_{1}, \ldots, \varepsilon_{n}\right)=\left\|B\left(n^{-1 / 2} \sigma^{-1} W_{n}\right)\right\|_{\alpha}$ converges in distribution to $\|B(W)\|_{\alpha}=T_{\alpha, \infty}(W)$. In view of (15) in Lemma 2.1, $n^{-1 / 2+\alpha} \sigma^{-1} \widehat{T}_{\alpha, n}$ converges in distribution to the same limit.

In the case of $p$-regularly varying innovations with $p>2$, in view of the the inclusion $\mathrm{RV}_{p} \subset \mathcal{L}_{r, \infty}^{o}$ for $r<p$, the limit distribution of $\widehat{T}_{\alpha, n}$ is given by Theorem 2.2, where $p$ is replaced by $2 \leq r<p$ and $\alpha_{p}$ by $\alpha_{r}=1 / 2-1 / r$, the choice of an appropriate $r$ depending on the rate of $\gamma_{n}$. Moreover, if the slowly varying function $v$ of Definition 1.2 tends to zero at infinity then $\mathrm{RV}_{p} \subset \mathcal{L}_{r, \infty}^{o}$ and Theorem 2.2 applies directly. 
Theorem 2.3. Assume that the $\varepsilon_{i}$ 's are mean zero i.i.d. random variables in $\mathrm{RV}_{p}$ for some $p \geq 2$ and the $y_{n, k}$ 's satisfy (9) with $\phi_{n}$ satisfying (10). Then for any $\alpha \in\left(\alpha_{p}, 1\right]$,

$$
b_{n}^{-1} \widehat{T}_{\alpha, n} \underset{n \rightarrow \infty}{\stackrel{\mathcal{D}}{\longrightarrow}} T_{p},
$$

where $T_{p}$ is a random variable with Fréchet distribution $\mathbb{P}\left(T_{p} \leq x\right)=\exp \left(-x^{-p}\right)$, $x>0$.

Proof. By Theorem 1.1. in [11], if the innovations $\varepsilon_{i}$ are i.i.d. and in $\mathrm{RV}_{p}$, then

$$
b_{n}^{-1} T_{\alpha, n}\left(\varepsilon_{1}, \ldots, \varepsilon_{n}\right) \underset{n \rightarrow \infty}{\stackrel{\mathcal{D}}{\longrightarrow}} T_{p},
$$

so (18) obviously follows from (12) in lemma 2.1.

\section{Consistency of test statistics}

In this section we investigate the consistency of the test statistics $\widehat{T}_{\alpha, n}$. So we are given a sample $\left(y_{n, k}, k=1, \ldots, n\right)$ generated from the first order autoregressive process with epidemic drift

$$
y_{n, k}=\phi_{n} y_{n, k-1}+\varepsilon_{k}+a_{n} \mathbf{1}_{\mathbb{I}_{n}^{*}}(k), \quad k=1, \ldots, n, \quad n \geq 1, \quad y_{n, 0}=0
$$

where $\mathbb{I}_{n}^{*}=\left\{k_{n}^{*}+1, \ldots, k_{n}^{*}+\ell_{n}^{*}\right\}$. Let us introduce

$$
\tau_{n, k}=\sum_{j=1}^{k} \phi_{n}^{k-j} a_{n, j}
$$

and

$$
z_{n, k}=y_{n, k}-\tau_{n, k}, \quad k=0,1, \ldots, n .
$$

Noting that $\tau_{n, k}-\phi_{n} \tau_{n, k-1}=a_{n, k}$, we can recast the model giving the $y_{n, k}$ 's as

$$
y_{n, k}-\tau_{n, k}=\phi_{n}\left(y_{n, k-1}-\tau_{n, k-1}\right)+\varepsilon_{k} .
$$

It follows that if $\left(y_{n, k}\right)$ satisfies $H_{A}$, then $\left(z_{n, k}\right)$ is an $A R(1)$ process satisfying $H_{0}$.

To exploit this feature, we can express the residuals in the following way.

$$
\begin{aligned}
\widehat{\varepsilon}_{k}=y_{n, k}-\widehat{\phi}_{n} y_{n, k-1} & =\phi_{n} y_{n, k-1}+\varepsilon_{k}+a_{n, k}-\widehat{\phi}_{n} y_{n, k-1} \\
& =\left(\phi_{n}-\widehat{\phi}_{n}\right)\left(z_{n, k-1}+\tau_{n, k-1}\right)+\varepsilon_{k}+a_{n, k} \\
& =a_{n, k}+\left(\phi_{n}-\widehat{\phi}_{n}\right) \tau_{n, k-1}+\left(\phi_{n}-\widehat{\phi}_{n}\right) z_{n, k-1}+\varepsilon_{k} .
\end{aligned}
$$


Triangle inequality applied to $\widehat{T}_{\alpha, n}=T_{\alpha, n}\left(\widehat{\varepsilon}_{1}, \ldots, \widehat{\varepsilon}_{n}\right)$ leads to

$$
\begin{aligned}
\widehat{T}_{\alpha, n} \geq & T_{\alpha, n}\left(a_{n, 1}, \ldots, a_{n, n}\right)-\left|\widehat{\phi}_{n}-\phi_{n}\right| T_{\alpha, n}\left(\tau_{n, 0}, \ldots, \tau_{n, n-1}\right) \\
& -\left|\widehat{\phi}_{n}-\phi_{n}\right| T_{\alpha, n}\left(z_{n, 0}, \ldots, z_{n, n-1}\right)-T_{\alpha, n}\left(\varepsilon_{1}, \ldots, \varepsilon_{n}\right) .
\end{aligned}
$$

Now, to obtain the consistency of our statistics $\widehat{T}_{\alpha, n}$, it suffices to prove that with the normalization already used under $H_{0}$, all the random terms in the above lower bound are negligible in probability when compared with the deterministic term $T_{\alpha, n}\left(a_{n, 1}, \ldots, a_{n, n}\right)$ which has to tend to infinity. In this way, it is convenient to replace $T_{\alpha, n}\left(a_{n, 1}, \ldots, a_{n, n}\right)$ by the following lower bound, assuming without loss of generality that $\ell_{n}^{*} \leq n / 2$ (recall we are looking for short epidemics).

$$
\begin{aligned}
T_{\alpha, n}\left(a_{n, 1}, \ldots, a_{n, n}\right) & =\max _{1 \leq \ell \leq n} \ell^{-\alpha} \max _{1 \leq k \leq n-\ell}\left|\sum_{j=k+1}^{k+\ell} a_{n} \mathbf{1}_{\mathbb{I}_{n}^{*}}(j)-\frac{l}{n} \sum_{j=1}^{n} a_{n} \mathbf{1}_{\mathbb{I}_{n}^{*}}(j)\right| \\
& \geq\left|a_{n}\right| \ell^{*(1-\alpha)}\left(1-\frac{\ell^{*}}{n}\right) \geq \frac{1}{2}\left|a_{n}\right| \ell^{*(1-\alpha)}
\end{aligned}
$$

As in (22), both random terms $T_{\alpha, n}\left(z_{n, 0}, \ldots, z_{n, n-1}\right)$ and $T_{\alpha, n}\left(\varepsilon_{1}, \ldots, \varepsilon_{n}\right)$ can be controlled by the Hölderian functional central limit theorems already used under $H_{0}$, it remains to find suitable estimates for $T_{\alpha, n}\left(\tau_{n, 0}, \ldots, \tau_{n, n-1}\right)$ and $\left|\widehat{\phi}_{n}-\phi_{n}\right|$. This is provided by the following lemmas, whose the quite technical proofs are postponed to Section B in the annex.

Lemma 3.1. Suppose that $k^{*} \geq \lambda n$ with some fixed $0<\lambda<1$. Assume that the innovations $\varepsilon_{i}$ of the process $\left(y_{n, k}\right)$ defined by (1) are square integrable and that $\gamma_{n}$ is increasing in $n$ or regularly varying. Then

$$
\left|\widehat{\phi}_{n}-\phi_{n}\right|=o_{P}\left(1-\phi_{n}\right)
$$

provided that

$$
a_{n}^{2} \ell^{*}=o\left(n\left(1-\phi_{n}\right)\right) .
$$

Lemma 3.2. Under $H_{A}$, with $\tau_{n, k}$ defined by (20),

$$
T_{\alpha, n}\left(\tau_{n, 0}, \ldots, \tau_{n, n-1}\right) \leq \frac{5\left|a_{n}\right|}{1-\phi_{n}} \ell^{*(1-\alpha)}
$$

Now we are in a position to give our consistency results. 
Theorem 3.3. Suppose that in the model defined by (1), $\phi_{n}$ is a constant $\phi \in(-1,1)$ and that the $\varepsilon_{i}$ are in $\mathcal{L}_{2}$ (then $p=2$ ) or in $\mathcal{L}_{p, \infty}^{o}$ for some $p>2$. Assume that

$$
\ell^{*} a_{n}^{2}=o(n)
$$

and that for some $\alpha \in\left[0, \alpha_{p}\right]$,

$$
n^{-1 / 2+\alpha}\left|a_{n}\right| \ell^{*(1-\alpha)} \rightarrow \infty .
$$

Then, under $H_{A}$,

$$
n^{-1 / 2+\alpha} \widehat{T}_{\alpha, n} \underset{n \rightarrow \infty}{\stackrel{\mathrm{P}}{\longrightarrow}} \infty
$$

Of course the same result holds when the $\varepsilon_{i}$ are in $\mathrm{RV}_{p}$, assuming that $\alpha<$ $\alpha_{p}$ in (27). It is worth noting here that if $a_{n}$ is constant, then Theorem 3.3 allows detection of short epidemics satisfying $n^{\beta}=o\left(\ell^{*}\right)$ with $\beta=(1 / 2-$ $\alpha) /(1-\alpha)$. In particular, if the $\varepsilon_{i}$ have finite $p$ moments for every $p>0$, then epidemics of length $n^{\beta}$ are detectable for arbitrarily small $\beta$. Since the proof of Theorem 3.3 is a simple adaptation of the one of Theorem 3.4 below, we omit it.

Theorem 3.4. Suppose that in the model defined by (1), the $\varepsilon_{i}$ 's are in $\mathcal{L}_{2}$ (then $p=2$ ) or in $\mathcal{L}_{p, \infty}^{o}$ for some $p>2$. Suppose moreover that $\phi_{n} \in(0,1)$, $\phi_{n} \rightarrow 1$ and that $\gamma_{n}=n\left(1-\phi_{n}\right)$ is non decreasing or regularly varying and satisfies (13). Under $H_{A}$, assume that

$$
\widehat{\phi}_{n}-\phi_{n}=o_{P}\left(1-\phi_{n}\right)
$$

that $\ell^{*} \rightarrow \infty, \ell^{*}=o(n)$ and for some $\alpha \in\left[0, \alpha_{p}\right]$,

$$
n^{-1 / 2+\alpha} \ell^{*(1-\alpha)}\left|a_{n}\right| \underset{n \rightarrow \infty}{\longrightarrow} \infty .
$$

Then

$$
n^{-1 / 2+\alpha} \widehat{T}_{\alpha, n} \underset{n \rightarrow \infty}{\stackrel{P}{\longrightarrow}} \infty
$$

Condition (28) holds in particular if $a_{n}^{2} \ell^{*}=o\left(n\left(1-\phi_{n}\right)\right)$.

Proof. As already seen in the proof of Theorem 2.2, the membership of $\varepsilon_{1}$ in $\mathcal{L}_{2}$ or in $\mathcal{L}_{p, \infty}^{o}$ implies the convergence in distribution of $n^{-1 / 2+\alpha} \sigma^{-1} T_{\alpha, n}\left(\varepsilon_{1}, \ldots, \varepsilon_{n}\right)$ to $T_{\alpha, \infty}(W)$ for every $\alpha \in\left[0, \alpha_{p}\right]$, with $\alpha_{2}=0$ in the $\mathcal{L}_{2}$ case, whence

$$
n^{-1 / 2+\alpha} T_{\alpha, n}\left(\varepsilon_{1}, \ldots, \varepsilon_{n}\right)=O_{P}(1) .
$$


Next, recalling that under $H_{A},\left(z_{n, k}\right)$ is a nearly non stationary process satisfying $H_{0}$, we know from (14) in Lemma 2.1 that for every $\alpha \in\left[0, \alpha_{p}\right]$,

$$
n^{-1 / 2+\alpha} T_{\alpha, n}\left(z_{n, 0}, \ldots, z_{n, n-1}\right)=O_{P}\left(1 /\left(1-\phi_{n}\right)\right) .
$$

Looking back at (22)-(25) and accounting (30), (31), we obtain the lower bound

$$
n^{-1 / 2+\alpha} \widehat{T}_{\alpha, n} \geq \frac{1}{2} n^{-1 / 2+\alpha}\left|a_{n}\right| \ell^{*(1-\alpha)}-\Delta_{n},
$$

where

$$
\Delta_{n}=5 n^{-1 / 2+\alpha}\left|a_{n}\right| \ell^{*(1-\alpha)} \frac{\left|\hat{\phi}_{n}-\phi_{n}\right|}{1-\phi_{n}}+O_{p}\left(1+\frac{\left|\widehat{\phi}_{n}-\phi_{n}\right|}{1-\phi_{n}}\right) .
$$

It is clear from this lower bound, that if $n^{-1 / 2+\alpha}\left|a_{n}\right| \ell^{*(1-\alpha)}$ tends to infinity and $\widehat{\phi}_{n}-\phi_{n}=o_{P}\left(1-\phi_{n}\right)$, then $n^{-1 / 2+\alpha} \widehat{T}_{\alpha, n}$ tends in probability to infinity. A concrete condition on $a_{n}^{2} \ell^{*}$ to have $\widehat{\phi}_{n}-\phi_{n}=o_{P}\left(1-\phi_{n}\right)$ is given by Lemma 3.1.

We complete Theorem 3.4 by treating the case $\alpha \in\left(\alpha_{p}, 1\right]$ for $\varepsilon_{i} \in \mathrm{RV}_{p}$.

Theorem 3.5. Suppose that in the model defined by (1), the $\varepsilon_{i}$ 's are in $\operatorname{RV}_{p}$ for some $p \geq 2$. Suppose moreover that $\phi_{n} \in(0,1), \phi_{n} \rightarrow 1$ and that $\gamma_{n}=n\left(1-\phi_{n}\right)$ is non decreasing or regularly varying, tends to infinity. Under $H_{A}$, assume that $\ell^{*} \rightarrow \infty, \ell^{*}=o(n)$ and

$$
b_{n}^{-1} \ell^{*(1-\alpha)}\left|a_{n}\right| \underset{n \rightarrow \infty}{\longrightarrow} \infty
$$

with $b_{n}$ defined by (6). If in addition $\widehat{\phi}_{n}$ satisfies (28), then

$$
b_{n}^{-1} \widehat{T}_{\alpha, n} \underset{n \rightarrow \infty}{\stackrel{\mathrm{P}}{\longrightarrow}} \infty
$$

Proof. The proof relies again on the lower bound (22), where the estimates (23) and (25) for the deterministic terms $T_{\alpha, n}\left(a_{n, 1}, \ldots, a_{n, n}\right)$ and $T_{\alpha, n}\left(\tau_{n, 0}, \ldots, \tau_{n, n-1}\right)$ remain valid. For the control of the random terms $T_{\alpha, n}\left(z_{n, 0}, \ldots, z_{n, n-1}\right)$, $T_{\alpha, n}\left(\varepsilon_{n, 0}, \ldots, \varepsilon_{n, n-1}\right)$, as the $\varepsilon_{i}$ 's are in $\mathrm{RV}_{p}$, Theorem 1.1. in [11] provides

$$
b_{n}^{-1} T_{\alpha, n}\left(\varepsilon_{1}, \ldots, \varepsilon_{n}\right)=O_{P}(1) .
$$

From (33), (11) in Lemma 2.1 applied to the process $\left(z_{n, k}\right)$ which satisfies $H_{0}$, and (28), we have

$$
\left|\widehat{\phi}_{n}-\phi_{n}\right| T_{\alpha, n}\left(z_{n, 0}, \ldots, z_{n, n-1}\right)=o_{P}\left(b_{n}\right) \text {. }
$$


Collecting all previous estimates we obtain from (22)

$$
\widehat{T}_{\alpha, n} \geq \frac{1}{2}\left|a_{n}\right| \ell^{*(1-\alpha)}-o_{P}\left(\left|a_{n}\right| \ell^{*(1-\alpha)}\right)-o_{p}\left(b_{n}\right)-O_{P}\left(b_{n}\right) .
$$

Clearly now, in order that $b_{n}^{-1} \widehat{T}_{\alpha, n}$ tends to infinity in probability, it suffices that $b_{n}^{-1}\left|a_{n}\right| \ell^{*(1-\alpha)}$ tends to infinity.

\section{A Proof of Lemma 2.1}

\section{A.1 Some useful inequalities}

The forthcoming proof of lemma 2.1, which is an essential tool in proving theorems 2.3 and 3.5, exploits intensively the following version of Hájek-Rényi inequality.

Lemma A.1. For each $n \geq 1$ let $\left(S_{n, k}, 1 \leq k \leq n\right)$ be a sequence of random variables, let $\left(\mu_{n, k}, 1 \leq k \leq n\right),\left(\nu_{n, k}, 1 \leq k \leq n\right)$ be sequences of nonnegative real numbers and let $r \geq 2$. If there exists $c>0$ such that for any $1 \leq m \leq n$ and any $\delta>0$

$$
\mathbb{P}\left(\max _{k \leq m}\left|S_{n, k}\right| \geq \delta\right) \leq c \delta^{-r}\left[\left(\sum_{k=1}^{m} \mu_{n, k}\right)^{r / 2}+\sum_{k=1}^{m} \nu_{n, k}\right]
$$

then for any sequence $\left(\beta_{n, k}, 1 \leq k \leq n\right)$ such that $0<\beta_{n, 1} \leq \cdots \leq \beta_{n, n}$ and any $\delta>0$ it holds

$$
\mathbb{P}\left(\max _{k \leq n} \beta_{n, k}^{-1}\left|S_{n, k}\right| \geq \delta\right) \leq 2^{1+r / 2} c \delta^{-r}\left[\left(\sum_{k=1}^{n} \beta_{n, k}^{-2} \mu_{n, k}\right)^{r / 2}+\sum_{k=1}^{n} \beta_{n, k}^{-r} \nu_{n, k}\right] .
$$

Proof. The proof is based on the idea of the proof of Theorem 1.1 in Fazekas and Klesov [12]. Without loss of generality we can assume that $\beta_{n, 1}=1$. Set for $i \geq 0$,

$$
A_{i}:=\left\{m: 1 \leq m \leq n \quad \text { and } \quad 2^{i} \leq \beta_{n, m}^{r}<2^{i+1}\right\}
$$

and let $I=\max \left\{i: A_{i} \neq \emptyset\right\}$. Let $m_{i}$ be a maximal element of the set $A_{i}$ and put $m_{i}=m_{i-1}$ if the set $A_{i}$ is empty. Then we have

$$
\begin{aligned}
\mathbb{P}\left(\max _{1 \leq k \leq n} \beta_{n, k}^{-1}\left|S_{n, k}\right|>\delta\right) & \leq \mathbb{P}\left(\max _{0 \leq i \leq I} 2^{-i / r} \max _{k \in A_{i}}\left|S_{n, k}\right| \geq \delta\right) \\
& \leq \sum_{i=0}^{I} \mathbb{P}\left(\max _{k \leq m_{i}}\left|S_{n, k}\right| \geq \delta 2^{i / r}\right) \\
& \leq c \delta^{-r} \sum_{i=0}^{I} 2^{-i}\left[\left(\sum_{k=1}^{m_{i}} \mu_{n, k}\right)^{r / 2}+\sum_{k=1}^{m_{i}} \nu_{n, k}\right]
\end{aligned}
$$


By comparison of $\ell^{p}$ and $\ell^{1}$ norms on $\mathbb{R}^{1+I}$ with $p=r / 2 \geq 1$,

$$
\sum_{i=0}^{I} 2^{-i}\left(\sum_{k=1}^{m_{i}} \mu_{n, k}\right)^{r / 2} \leq\left(\sum_{i=0}^{I} 2^{-2 i / r} \sum_{k=1}^{m_{i}} \mu_{n, k}\right)^{r / 2}
$$

from which we deduce

$$
\mathbb{P}\left(\max _{1 \leq k \leq n} \beta_{k}^{-1}\left|S_{n, k}\right|>\delta\right) \leq c \delta^{-r}\left[\left(\sum_{i=0}^{I} 2^{-2 i / r} \sum_{k=1}^{m_{i}} \mu_{n, k}\right)^{r / 2}+\sum_{i=0}^{I} 2^{-i} \sum_{k=1}^{m_{i}} \nu_{n, k}\right] .
$$

Changing the summation according to the scheme

$\sum_{i=0}^{I} d_{i} \sum_{k=1}^{m_{i}} u_{k}=\sum_{i=0}^{I} \sum_{j=0}^{I} \sum_{k \in A_{j}} d_{i} u_{k} \mathbf{1}_{\{j \leq i\}}=\sum_{j=0}^{I} \sum_{k \in A_{j}} \sum_{i=0}^{I} d_{i} u_{k} \mathbf{1}_{\{j \leq i\}}=\sum_{j=0}^{I} \sum_{k \in A_{j}} u_{k} \sum_{i=j}^{I} d_{i}$

we obtain

$$
\begin{aligned}
\mathbb{P}\left(\max _{1 \leq k \leq n} \beta_{k}^{-1}\left|S_{n, k}\right|>\delta\right) & \leq c \delta^{-r}\left[\left(\sum_{j=0}^{I} \sum_{k \in A_{j}} \mu_{n, k} \sum_{i=j}^{I} 2^{-2 i / r}\right)^{r / 2}+\sum_{j=0}^{I} \sum_{k \in A_{j}} \nu_{n, k} \sum_{i=j}^{I} 2^{-i}\right] \\
& \leq 2^{r / 2} c \delta^{-r}\left[\left(\sum_{j=0}^{I} \sum_{k \in A_{j}} 2^{-2 j / r} \mu_{n, k}\right)^{r / 2}+\sum_{j=0}^{I} \sum_{k \in A_{j}} 2^{-j} \nu_{n, k}\right] \\
& \leq 2^{1+r / 2} c \delta^{-r}\left[\left(\sum_{j=0}^{I} \sum_{k \in A_{j}} \beta_{n, k}^{-2} \mu_{n, k}\right)^{r / 2}+\sum_{j=0}^{I} \sum_{k \in A_{j}} \beta_{n, k}^{-r} \nu_{n, k}\right] \\
& =2^{1+r / 2} c \delta^{-r}\left[\left(\sum_{k=1}^{n} \beta_{n, k}^{-2} \mu_{n, k}\right)^{r / 2}+\sum_{k=1}^{n} \beta_{n, k}^{-r} \nu_{n, k}\right]
\end{aligned}
$$

and the result is proved.

We will need also the following inequality for which we refer to [9, Lemma $2]$.

Lemma A.2. Let $\left(\eta_{j}\right)_{j \geq 0}$ be a sequence of i.i.d. random variables with $\mathbb{E} \eta_{0}=$ 0 and $\mathbb{E}\left|\eta_{0}\right|^{q}<\infty$ for some $q \geq 2$. Suppose that $\phi_{n} \rightarrow 1$ and $n\left(1-\phi_{n}\right) \rightarrow \infty$ as $n \rightarrow \infty$. Then there exists an integer $n_{0}(q) \geq 1$, depending on $q$ only, such that, for all $n \geq n_{0}(q)$, and $\lambda>0$,

$$
\mathbb{P}\left(\max _{1 \leq k \leq n}\left|\sum_{j=1}^{k} \phi_{n}^{k-j} \eta_{j}\right|>\lambda\right) \leq 4 C_{q} \mathrm{e}^{q} \lambda^{-q} n^{q / 2} \mathbb{E}\left|\eta_{0}\right|^{q}\left(n\left(1-\phi_{n}\right)\right)^{1-q / 2},
$$

where $C_{q}$ is the universal constant in the Rosenthal inequality of order $q$.

Finally the following estimates for truncated moments will be useful. 
Lemma A.3. Let $X$ be a non negative random variable.

a) Assume that $X \in \mathcal{L}_{p, \infty}$ for some $p>1$ and put $N_{p}(X):=\sup _{t>0} t^{p} \mathbb{P}(X>$ t). Then for every $u>0$,

$$
\mathbb{E}\left(X \mathbf{1}_{\{X \geq u\}}\right) \leq \frac{p}{p-1} N_{p}(X) u^{1-p}
$$

and for every $q>p$,

$$
\mathbb{E}\left(X \mathbf{1}_{\{X \leq u\}}\right)^{q} \leq \frac{q}{q-p} N_{p}(X) u^{q-p} .
$$

b) Assume that $\mathbb{P}(X>t)$ is regularly varying with index $-p$ (this condition is satisfied in particular when $\left.X \in \operatorname{RV}_{p}\right)$. Let $b_{n}=\inf \{t>0: \mathbb{P}(X \leq$ $t) \geq 1-1 / n\}$. Then for any $\delta>0$,

$$
\mathbb{E}\left(X \mathbf{1}_{\left\{X \geq h b_{n}\right\}}\right) \leq \frac{p(1+\delta)}{p-1} h^{1-p} b_{n} n^{-1},
$$

for $n$ large enough, uniformly in $h \in[1, \infty)$. For any $r>p$, any $\delta>0$,

$$
\mathbb{E}\left(X \mathbf{1}_{\left\{X \leq h b_{n}\right\}}\right)^{r} \leq \frac{r(1+\delta)}{r-p} h^{r-p} b_{n}^{r} n^{-1},
$$

for $n$ large enough, uniformly in $h \in[1, \infty)$.

Proof. To prove (35) we observe that $\mathbb{P}\left(X 1_{\{X \geq u\}}>t\right)=\mathbb{P}(X>\max (t, u))$, for any $t>0$, whence

$$
\begin{aligned}
\mathbb{E}\left(X 1_{\{X \geq u\}}\right)=\int_{0}^{\infty} \mathbb{P}\left(X 1_{\{X \geq u\}}>t\right) \mathrm{d} t & =\int_{0}^{u} \mathbb{P}(X>u) \mathrm{d} t+\int_{u}^{\infty} \mathbb{P}(X>t) \mathrm{d} t \\
& \leq u \mathbb{P}(X>u)+\int_{u}^{\infty} \frac{N_{p}(X)}{t^{p}} \mathrm{~d} t \\
& \leq N_{p}(X) u^{1-p}+N_{p}(X) \frac{u^{1-p}}{p-1}=\frac{p N_{p}(X)}{p-1} u^{1-p} .
\end{aligned}
$$

To prove (36), we note that for $t, u>0, \mathbb{P}\left(X 1_{\{X \leq u\}}>t\right)=\mathbb{P}(t<X \leq$ $u$ ), whence

$$
\begin{aligned}
\mathbb{E}\left(X \mathbf{1}_{\{X \leq u\}}\right)^{q}=\int_{0}^{\infty} q t^{q-1} \mathbb{P}\left(X \mathbf{1}_{\{X \leq u\}}>t\right) \mathrm{d} t & =\int_{0}^{\infty} q t^{q-1} \mathbb{P}(t<X \leq u) \mathrm{d} t \\
& =\int_{0}^{u} q t^{q-1} \mathbb{P}(t<X \leq u) \mathrm{d} t \\
& \leq \int_{0}^{u} q t^{q-1} \mathbb{P}(X>t) \mathrm{d} t \\
& \leq \int_{0}^{u} q t^{q-p-1} N_{p}(X) \mathrm{d} t=\frac{q N_{p}(X)}{q-p} u^{q-p} .
\end{aligned}
$$


The proof of (37) starts like the one of (35):

$$
\mathbb{E}\left(X \mathbf{1}_{\left\{X \geq h b_{n}\right\}}\right)=h b_{n} \mathbb{P}\left(X>h b_{n}\right)+\int_{h b_{n}}^{\infty} \mathbb{P}(X>t) \mathrm{d} t
$$

Now, as $\mathbb{P}(X>t)$ is regularly varying with index $-p$, by Prop. 1.5.10 in [8],

$$
\frac{h b_{n} \mathbb{P}\left(X>h b_{n}\right)}{\int_{h b_{n}}^{\infty} \mathbb{P}(X>t) \mathrm{d} t} \underset{n \rightarrow \infty}{\longrightarrow} p-1, \quad \text { uniformly in } h \geq 1
$$

which combined with (39) gives

$$
\mathbb{E}\left(X \mathbf{1}_{\left\{X \geq h b_{n}\right\}}\right) \sim \frac{p}{p-1} h b_{n} \mathbb{P}\left(X>h b_{n}\right), \quad \text { uniformly in } h \geq 1 .
$$

By th. 1.5.2 in [8],

$$
\mathbb{P}\left(X>h b_{n}\right) \sim h^{-p} \mathbb{P}\left(X>b_{n}\right), \quad \text { uniformly in } h \geq 1 .
$$

From the definition of the quantile $b_{n}, \mathbb{P}\left(X>b_{n}\right) \leq n^{-1}$. Combining this estimate with (40) and (41) gives (37).

To prove (38), we begin by noting that like in the proof of (36),

$$
\mathbb{E}\left(X \mathbf{1}_{\left\{X \leq h b_{n}\right\}}\right)^{r} \leq \int_{0}^{h b_{n}} r t^{r-1} \mathbb{P}(X>t) \mathrm{d} t .
$$

Next, by Th. 1.5.11 (i) in [8],

$$
\frac{\left(h b_{n}\right)^{r} \mathbb{P}\left(X>h b_{n}\right)}{\int_{0}^{h b_{n}} t^{r-1} \mathbb{P}(X>t) \mathrm{d} t} \underset{n \rightarrow \infty}{\longrightarrow} r-p, \quad \text { uniformly in } h \geq 1,
$$

from which we obtain (38) in the same way as for (37).

\section{A.2 Proof of Lemma 2.1, common part}

Since

$$
\sum_{j=k+1}^{k+\ell} y_{n, j-1}=\frac{1}{1-\phi_{n}}\left[\sum_{j=k+1}^{k+\ell} \varepsilon_{j}+y_{n, k}-y_{n, k+\ell}\right]
$$

and

$$
y_{n, k+\ell}-y_{n, k}=\sum_{j=1}^{k+\ell} \phi_{n}^{k+\ell-j} \varepsilon_{j}-\sum_{j=1}^{k} \phi_{n}^{k-j} \varepsilon_{j}=\sum_{j=k+1}^{k+\ell} \phi_{n}^{k+\ell-j} \varepsilon_{j}-\left(1-\phi_{n}^{\ell}\right) y_{n, k}
$$

for any $1 \leq \ell \leq n, 0 \leq k \leq n-\ell$ and $y_{n, 0}=0$, we deduce

$$
\left|\left(1-\phi_{n}\right) T_{\alpha, n}\left(y_{n, 0}, \ldots, y_{n, n-1}\right)-T_{\alpha, n}\left(\varepsilon_{1}, \ldots, \varepsilon_{n}\right)\right| \leq T_{n}^{(1)}+T_{n}^{(2)}+T_{n}^{(3)},
$$


where

$$
\begin{aligned}
T_{n}^{(1)} & :=\max _{1 \leq \ell<n} \ell^{-\alpha} \max _{1 \leq k \leq n-\ell}\left|\sum_{j=k+1}^{k+\ell} \phi_{n}^{k+\ell-j} \varepsilon_{j}\right|, \\
T_{n}^{(2)} & :=\max _{1 \leq \ell<n} \ell^{-\alpha} \max _{1 \leq k \leq n-\ell}\left|\left(1-\phi_{n}^{\ell}\right) y_{n, k}\right| \\
T_{n}^{(3)} & =\max _{1 \leq \ell<n} \ell^{-\alpha}(\ell / n)\left|y_{n, n}\right|=n^{-\alpha}\left|y_{n, n}\right| .
\end{aligned}
$$

We will show separately below that in the case a) of Lemma 2.1

$$
T_{n}^{(i)}=O_{P}\left(b_{n}\right), \quad i=1,2,3,
$$

while in case b)

$$
T_{n}^{(i)}=O_{P}\left(n^{1 / 2-\alpha}\right), \quad i=1,2,3 .
$$

This will establish (11) and (14).

Before this splitting of the proof, we can already note that (12) and (15) respectively follow from (11) and (14). Indeed for (12) we observe that

$$
\left|T_{\alpha, n}\left(\widehat{\varepsilon}_{1}, \ldots, \widehat{\varepsilon}_{n}\right)-T_{\alpha, n}\left(\varepsilon_{1}, \ldots, \varepsilon_{n}\right)\right| \leq\left|\widehat{\phi}_{n}-\phi_{n}\right| T_{\alpha, n}\left(y_{n, 0}, \ldots, y_{n, n}\right) .
$$

Since $T_{\alpha, n}\left(\varepsilon_{1}, \ldots, \varepsilon_{n}\right)=O_{P}\left(b_{n}\right)$ by Theorem 1.1. in [11], (11) gives

$$
\left(1-\phi_{n}\right) T_{\alpha, n}\left(y_{n, 0}, \ldots, y_{n, n}\right)=O_{P}\left(b_{n}\right) .
$$

By Giraitis and Philips [7, Th. 1], $n^{1 / 2}\left(1-\phi_{n}^{2}\right)^{-1 / 2}\left(\widehat{\phi}_{n}-\phi_{n}\right)$ is asymptoticaly normal provided that $\mathbb{E} \varepsilon_{1}^{2}<\infty$, so

$$
\widehat{\phi}_{n}-\phi_{n}=O_{P}\left(\frac{\left(1-\phi_{n}\right)^{1 / 2}}{n^{1 / 2}}\right)
$$

We then deduce from (44) that

$$
\begin{aligned}
T_{\alpha, n}\left(\widehat{\varepsilon}_{1}, \ldots, \widehat{\varepsilon}_{n}\right)-T_{\alpha, n}\left(\varepsilon_{1}, \ldots, \varepsilon_{n}\right) & =O_{P}\left(\frac{b_{n}\left|\widehat{\phi}_{n}-\phi_{n}\right|}{1-\phi_{n}}\right) \\
& =O_{P}\left(\frac{b_{n}}{n^{1 / 2}\left(1-\phi_{n}\right)^{1 / 2}}\right)=o_{P}\left(b_{n}\right) .
\end{aligned}
$$

since $n\left(1-\phi_{n}\right)$ tends to infinity.

The deduction of (15) from (14) is essentially the same. We just have to replace $b_{n}$ by $n^{1 / 2-\alpha}$ and note that the estimate $T_{\alpha, n}\left(\varepsilon_{1}, \ldots, \varepsilon_{n}\right)=O_{P}\left(n^{1 / 2-\alpha}\right)$ is a by-product of the convergence in distribution of $n^{-1 / 2+\alpha} T_{\alpha, n}\left(\varepsilon_{1}, \ldots, \varepsilon_{n}\right)$ already established in the proof of Theorem 2.2. 


\section{A.3 Proof of Lemma 2.1, estimate (11)}

Estimate for $T_{n}^{(1)}$. For $h>0$ set

$$
P_{n, h}^{(1)}:=\mathbb{P}\left(T_{n}^{(1)}>2 h b_{n}\right) .
$$

To estimate this probability we define the truncated random variables:

$$
\varepsilon_{j}^{\prime}=\varepsilon_{j} \mathbf{1}_{\left\{\left|\varepsilon_{j}\right|>h b_{n}\right\}}, \quad \varepsilon_{j}^{\prime \prime}=\varepsilon_{j} \mathbf{1}_{\left\{\left|\varepsilon_{j}\right| \leq h b_{n}\right\}}-\mathbb{E} \varepsilon_{j} \mathbf{1}_{\left\{\left|\varepsilon_{j}\right| \leq h b_{n}\right\}},
$$

for $j \geq 1$. Then

$$
P_{n, h}^{(1)} \leq P_{n, h}^{(1,1)}+P_{n, h}^{(1,2)}
$$

where

$$
P_{n, h}^{(1,1)}:=\mathbb{P}\left(\max _{1 \leq j \leq n}\left|\varepsilon_{j}\right|>h b_{n}\right), \quad P_{n, h}^{(1,2)}:=\mathbb{P}\left(T_{n}^{(1,2)}>2 h b_{n}\right)
$$

with

$$
T_{n}^{(1,2)}:=\max _{1 \leq \ell<n} \ell^{-\alpha} \max _{1 \leq k \leq n-\ell}\left|\sum_{j=k+1}^{k+\ell} \phi_{n}^{k+\ell-j} \varepsilon_{j} \mathbf{1}_{\left\{\left|\varepsilon_{j}\right| \leq h b_{n}\right\}}\right| .
$$

From extreme value theory we know (see, for example [13], Theorem 3.3.7) that

$$
\mathbb{P}\left(\max _{1 \leq j \leq n}\left|\varepsilon_{j}\right|>h b_{n}\right) \rightarrow 1-\exp \left\{-h^{-p}\right\}
$$

as $n \rightarrow \infty$. Choosing $h$ big enough we make probability $P^{(1,1)}$ arbitrary small, in other words

$$
\lim _{h \rightarrow \infty} \limsup _{n \rightarrow \infty} P_{n, h}^{(1,1)}=0 .
$$

Next we estimate $P_{n, h}^{(1,2)}$. First we need to center each $\varepsilon_{i} \mathbf{1}_{\left\{\left|\varepsilon_{i}\right| \leq h b_{n}\right\}}, i=$ $1, \ldots, n$. Observing that $\mathbb{E} \varepsilon_{i}=0, \mathbb{E} \varepsilon_{i} \mathbf{1}_{\left\{\left|\varepsilon_{i}\right| \leq h b_{n}\right\}}=\mathbb{E} \varepsilon_{i} \mathbf{1}_{\left\{\left|\varepsilon_{i}\right|>h b_{n}\right\}}$, we have

$$
\max _{1 \leq \ell \leq n} \ell^{-\alpha}\left|\sum_{j=k+1}^{k+\ell} \phi_{n}^{k+\ell-j} \mathbb{E}\left[\varepsilon_{j} \mathbf{1}_{\left\{\left|\varepsilon_{j}\right| \leq h b_{n}\right\}}\right]\right| \leq n^{1-\alpha} \mathbb{E}\left|\varepsilon_{1}\right| \mathbf{1}_{\left\{\left|\varepsilon_{1}\right| \geq h b_{n}\right\}} .
$$

By (37) in Lemma A.3,

$$
\mathbb{E}\left|\varepsilon_{1}\right| \mathbf{1}_{\left\{\left|\varepsilon_{1}\right| \geq h b_{n}\right\}} \leq 2 p(p-1)^{-1} n^{-1} b_{n} h^{1-p}
$$

for $n$ large enough uniformly in $h \geq 1$, we obtain

$$
\max _{1 \leq \ell \leq n} \ell^{-\alpha}\left|\sum_{j=k+1}^{k+\ell} \phi_{n}^{k+\ell-j} \mathbb{E}\left[\varepsilon_{j} \mathbf{1}_{\left\{\left|\varepsilon_{j}\right| \leq h b_{n}\right\}}\right]\right| \leq \frac{2 p}{(p-1) n^{\alpha} h^{p}} h b_{n} \leq h b_{n}
$$


for $n$ large enough uniformly in $h \geq 1$. It follows that for $n$ large enough and $h \geq 1$,

$$
P_{n, h}^{(1,2)} \leq \mathbb{P}\left(T_{n}^{(1,2,1)}>h b_{n}\right)
$$

with

$$
T_{n}^{(1,2,1)}:=\max _{1 \leq \ell<n} \ell^{-\alpha} \max _{1 \leq k \leq n-\ell}\left|\sum_{j=k+1}^{k+\ell} \phi_{n}^{k+\ell-j} \varepsilon_{j}^{\prime \prime}\right| .
$$

Since

$$
T_{n}^{(1,2,1)}=\max _{1 \leq \ell<n} \ell^{-\alpha} \max _{1 \leq k \leq n-\ell}\left|\sum_{j=1}^{\ell} \phi_{n}^{\ell-j} \varepsilon_{k+j}^{\prime \prime}\right| \leq \max _{1 \leq k \leq n} \max _{1 \leq \ell \leq n} \ell^{-\alpha}\left|\sum_{j=1}^{\ell} \phi_{n}^{\ell-j} \varepsilon_{k+j}^{\prime \prime}\right|
$$

we have

$$
\begin{aligned}
\mathbb{P}\left(T_{n}^{(1,2,1)} \geq h b_{n}\right) & \leq \sum_{k=1}^{n} \mathbb{P}\left(\max _{1 \leq \ell \leq n} \ell^{-\alpha}\left|\sum_{j=1}^{\ell} \phi_{n}^{\ell-j} \varepsilon_{k+j}^{\prime \prime}\right| \geq h b_{n}\right) \\
& =n \mathbb{P}\left(\max _{1 \leq \ell \leq n} \ell^{-\alpha}\left|\sum_{j=1}^{\ell} \phi_{n}^{\ell-j} \varepsilon_{j}^{\prime \prime}\right| \geq h b_{n}\right)
\end{aligned}
$$

due to stationarity. Choose $r>p$. Using successively Markov's, Doob's and Rosenthal's inequalities, we obtain for each $\delta>0$

$$
\mathbb{P}\left(\max _{1 \leq \ell \leq n}\left|\sum_{j=1}^{\ell} \phi_{n}^{-j} \varepsilon_{j}^{\prime \prime}\right|>\delta\right) \leq c \delta^{-r}\left[\left(\sum_{j=1}^{n} \phi_{n}^{-2 j} \mathbb{E}\left(\varepsilon_{1}^{\prime \prime}\right)^{2}\right)^{r / 2}+\sum_{j=1}^{n} \phi_{n}^{-r j} \mathbb{E}\left|\varepsilon_{1}^{\prime \prime}\right|^{r}\right]
$$

with a constant $c>0$ depending on $r$ only. Using (38) in Lemma A.3 together with the inequality $(|a|+|b|)^{r} \leq 2^{r-1}\left(|a|^{r}+|b|^{r}\right)$, we obtain

$$
\mathbb{E}\left|\varepsilon_{1}^{\prime \prime}\right|^{r} \leq 2^{r} \mathbb{E}\left(\left|\varepsilon_{1}\right| \mathbf{1}_{\left\{\left|\varepsilon_{1}\right| \leq h b_{n}\right\}}\right)^{r} \leq \frac{r 2^{r+1}}{r-p} h^{r-p} b_{n}^{r} n^{-1},
$$

for $n$ large enough, uniformly in $h \in[1, \infty)$. Hence, there is a constant $c>0$ depending on $r$ and $p$ only, such that for $n$ large enough and $h \geq 1$,

$$
\mathbb{P}\left(\max _{1 \leq \ell \leq n}\left|\sum_{j=1}^{\ell} \phi_{n}^{-j} \varepsilon_{j}^{\prime \prime}\right|>\delta\right) \leq c \delta^{-r}\left[\left(\sum_{k=1}^{n} \mu_{n, k}\right)^{r / 2}+\sum_{k=1}^{n} \nu_{n, k}\right],
$$

where $\mu_{n, k}=\sigma^{2} \phi_{n}^{-2 k}$ and $\nu_{n, k}=\phi_{n}^{-r k} h^{r-p} n^{-1} b_{n}^{r}, k=1, \ldots, n$. By lemma A.1 we deduce

$$
\begin{aligned}
\mathbb{P}\left(\max _{1 \leq \ell \leq n} \ell^{-\alpha}\left|\sum_{j=1}^{\ell} \phi_{n}^{\ell-j} \varepsilon_{j}^{\prime \prime}\right| \geq h b_{n}\right) & \leq c h^{-r} b_{n}^{-r}\left[\left(\sum_{k=1}^{n} k^{-2 \alpha} \phi_{n}^{2 k} \mu_{n, k}\right)^{r / 2}+\sum_{k=1}^{n} \phi_{n}^{r k} k^{-r \alpha} \nu_{n, k}\right] \\
& =c h^{-r} b_{n}^{-r}\left[\sigma^{r}\left(\sum_{k=1}^{n} k^{-2 \alpha}\right)^{r / 2}+\sum_{k=1}^{n} k^{-r \alpha} h^{r-p} n^{-1} b_{n}^{r}\right] .
\end{aligned}
$$


Since $\sum_{k=1}^{n} k^{-d \alpha}=O\left(\max \left\{n^{1-d \alpha}, 1\right\}\right)$ for $d>0$, choosing $r>1 / \alpha$ we have with some positive constant $C$,

$\mathbb{P}\left(\max _{1 \leq \ell \leq n} \ell^{-\alpha}\left|\sum_{j=1}^{\ell} \phi_{n}^{\ell-j} \varepsilon_{j}^{\prime \prime}\right| \geq h b_{n}\right) \leq C h^{-r} b_{n}^{-r}\left(\max \left\{n^{r(1-2 \alpha) / 2}, 1\right\}+h^{r-p} n^{-1} b_{n}^{r}\right)$.

Hence,

$$
\mathbb{P}\left(T_{n}^{(1,2,1)}>h b_{n}\right) \leq C\left(h^{-r} b_{n}^{-r} \max \left\{n, n^{1+r(1-2 \alpha) / 2}\right\}+h^{-p}\right) .
$$

By choosing $r>(1 / p-1 / 2+\alpha)^{-1}$ we see that

$$
\lim _{h \rightarrow \infty} \limsup _{n \rightarrow \infty} \mathbb{P}\left(T_{n}^{(1,2,1)} \geq h b_{n}\right)=0 .
$$

The proof of (42) for $i=1$ is now complete.

Estimate for $T_{n}^{(2)}$. First we note that

$$
T_{n}^{(2)}=\max _{1 \leq \ell<n} \ell^{-\alpha}\left(1-\phi_{n}^{\ell}\right) \max _{1 \leq k \leq n-\ell}\left|y_{n, k}\right| \leq\left(\max _{1 \leq \ell<n} \ell^{-\alpha}\left(1-\phi_{n}^{\ell}\right)\right) \max _{1 \leq j \leq n}\left|y_{n, j}\right|
$$

Then we observe that

$\max _{1 \leq \ell<n} \ell^{-\alpha}\left(1-\phi_{n}^{\ell}\right) \leq \sup _{t \geq 1} t^{-\alpha}\left(1-\phi_{n}^{t}\right)=\left|\ln \phi_{n}\right|^{\alpha} \sup _{u \geq\left|\ln \phi_{n}\right|} u^{-\alpha}\left(1-\mathrm{e}^{-u}\right) \leq\left|\ln \phi_{n}\right|^{\alpha}$.

Since $\left|\ln \phi_{n}\right|=\ln \left(1 / \phi_{n}\right) \leq \phi_{n}^{-1}-1$, we obtain

$$
\max _{1 \leq \ell<n} \ell^{-\alpha}\left(1-\phi_{n}^{\ell}\right) \leq \frac{\left(1-\phi_{n}\right)^{\alpha}}{\phi_{n}^{\alpha}}=O\left(\left(1-\phi_{n}\right)^{\alpha}\right) .
$$

Next we prove that

$$
\max _{1 \leq k \leq n}\left|y_{n, k}\right|=O_{P}\left(b_{n}\left(1-\phi_{n}\right)^{-\alpha}\right)
$$

To this aim we use similar techniques as above. Set for $h>0$

$$
P_{n}^{(2)}=\mathbb{P}\left(\max _{1 \leq k \leq n}\left|y_{n, k}\right|>2 h b_{n}\left(1-\phi_{n}\right)^{-\alpha}\right) .
$$

Then $P_{n}^{(2)} \leq P_{n}^{(2,1)}+P_{n}^{(2,2)}$, where

$$
\begin{aligned}
& P_{n}^{(2,1)}=\mathbb{P}\left(\max _{1 \leq k \leq n}\left|\varepsilon_{k}\right|>h b_{n}\right), \\
& P_{n}^{(2,2)}=\mathbb{P}\left(\max _{1 \leq k \leq n}\left|\sum_{j=1}^{k} \phi_{n}^{k-j} \varepsilon_{j} \mathbf{1}_{\left\{\left|\varepsilon_{j}\right| \leq h b_{n}\right\}}\right| \geq 2 h b_{n}\left(1-\phi_{n}\right)^{-\alpha}\right) .
\end{aligned}
$$


Since $P_{n}^{(2,1)}=P_{n}^{(1,1)}$ we have from (45)

$$
\lim _{h \rightarrow \infty} \limsup _{n \rightarrow \infty} P_{n}^{(2,1)}=0 .
$$

Since

$$
\max _{1 \leq k \leq n}\left|\sum_{j=1}^{k} \phi_{n}^{k-j} \mathbb{E} \varepsilon_{j} \mathbf{1}_{\left\{\left|\varepsilon_{j}\right| \leq h b_{n}\right\}}\right| \leq n \mathbb{E}\left|\varepsilon_{1}\right| \mathbf{1}_{\left\{\left|\varepsilon_{1}\right| \geq h b_{n}\right\}} \leq \frac{2 p}{p-1} b_{n} h^{1-p},
$$

using again (46), we deduce

$$
P_{n}^{(2,2)} \leq \mathbb{P}\left(\max _{1 \leq k \leq n}\left|\sum_{j=1}^{k} \phi_{n}^{k-j} \varepsilon_{j}^{\prime \prime}\right| \geq h b_{n}\left(1-\phi_{n}\right)^{-\alpha}\right)
$$

for $n$ large enough and $h \geq(2 p)^{1 / p}(p-1)^{-1 / p}$. Now we apply lemma A.2 and obtain

$$
P_{n}^{(2,2)} \leq c h^{-q} b_{n}^{-q}\left(1-\phi_{n}\right)^{q \alpha} \mathbb{E}\left|\varepsilon_{1}^{\prime \prime}\right|^{q} n^{q / 2}\left(n\left(1-\phi_{n}\right)\right)^{1-q / 2},
$$

with a constant depending only on $q$. Using (47) to bound $\mathbb{E}\left|\varepsilon_{1}^{\prime \prime}\right|^{q}$ we deduce

$$
P_{n}^{(2,2)} \leq \operatorname{ch}^{-p}\left(1-\phi_{n}\right)^{q \alpha+1-q / 2} .
$$

where $c$ depends on $p$ and $q$ only.

If $\alpha \geq 1 / 2$, then $1+q \alpha-q / 2>0$ and so

$$
\lim _{n \rightarrow \infty} P_{n}^{(2,2)}=0 \text {. }
$$

If $\alpha<1 / 2$ then $1+q \alpha-q / 2>0$ provided $q<1 /(1 / 2-\alpha)$. So we have to choose $p<q<1 /(1 / 2-\alpha)$ in the case $\alpha<1 / 2$. This is possible since $\alpha>1 / 2-1 / p$. So in any case (50) is valid and (42) with $i=2$ follows.

Estimate for $T_{n}^{(3)}$. We have $\mathbb{E} y_{n}^{2}=\sum_{k=1}^{n} \phi_{n}^{2(n-k)} \sigma^{2} \leq n \sigma^{2}$. Hence, $\left|y_{n}\right|=$ $O_{P}\left(n^{1 / 2}\right)$ and we obtain $T_{n}^{(3)}=n^{-\alpha} O_{P}\left(n^{1 / 2}\right)=o_{P}\left(b_{n}\right)$ recalling that $b_{n}=$ $n^{1 / p} v(n)$ with a slowly varying function $v(n)$ and observing that

$$
n^{-\alpha} n^{1 / 2} b_{n}^{-1}=n^{-\alpha-(1 / p)+(1 / 2)} v(n)^{-1} \rightarrow 0
$$

since $\alpha>1 / 2-1 / p$.

The proof of (11) is now complete. 


\section{A.4 Proof of Lemma 2.1, estimate (14)}

Next we consider the case (b) and prove (43) for $i=1,2,3$.

Estimate for $T_{n}^{(1)}$. Set $p_{\alpha}=1 /(1 / 2-\alpha)$. For $h>0$ set

$$
P_{n, h}^{(1)}:=\mathbb{P}\left(T_{n}^{(1)}>2 h n^{1 / p_{\alpha}}\right) .
$$

To estimate this probability we define the truncated random variables:

$$
\varepsilon_{j}^{\prime}=\varepsilon_{j} \mathbf{1}\left\{\left|\varepsilon_{j}\right|>h n^{1 / p_{\alpha}}\right\}, \quad \varepsilon_{j}^{\prime \prime}=\varepsilon_{j} \mathbf{1}\left\{\left|\varepsilon_{j}\right| \leq h n^{1 / p_{\alpha}}\right\}-\mathbb{E} \varepsilon_{j} \mathbf{1}\left\{\left|\varepsilon_{j}\right| \leq h n^{1 / p_{\alpha}}\right\},
$$

for $j \geq 1$. Then

$$
P_{n, h}^{(1)} \leq P_{n, h}^{(1,1)}+P_{n, h}^{(1,2)},
$$

where

$$
P_{n, h}^{(1,1)}:=\mathbb{P}\left(\max _{1 \leq j \leq n}\left|\varepsilon_{j}\right|>h n^{1 / p_{\alpha}}\right), \quad P_{n, h}^{(1,2)}:=\mathbb{P}\left(T_{n}^{(1,2)}>2 h n^{1 / p_{\alpha}}\right)
$$

with

$$
T_{n}^{(1,2)}:=\max _{1 \leq \ell<n} \ell^{-\alpha} \max _{1 \leq k \leq n-\ell}\left|\sum_{j=k+1}^{k+\ell} \phi_{n}^{k+\ell-j} \varepsilon_{j} \mathbf{1}_{\left\{\left|\varepsilon_{j}\right| \leq h n^{1 / p_{\alpha}}\right\}}\right| .
$$

Since

$$
\mathbb{P}\left(\max _{1 \leq j \leq n}\left|\varepsilon_{j}\right|>h n^{1 / 2-\alpha}\right) \leq n \mathbb{P}\left(\left|\varepsilon_{1}\right|>h n^{1 / 2-\alpha}\right),
$$

$P_{n, h}^{(1,1)}$ tends to 0 as $n \rightarrow \infty$ due to the condition $\varepsilon_{i} \in \mathcal{L}_{p, \infty}^{o}$ and $0 \leq \alpha \leq 1 / 2-$ $1 / p$ when $p>2$ or to the condition $\varepsilon_{i} \in \mathcal{L}_{2}$ when $p=2$ (then $\left.\alpha=\alpha_{2}=0\right)$.

Next we estimate $P_{n, h}^{(1,2)}$. First we need to center each $\varepsilon_{i} \mathbf{1}_{\left\{\left|\varepsilon_{i}\right| \leq h n^{1 / p} \alpha\right.}$, $i=1, \ldots, n$. From (35) we get that

$$
\mathbb{E}\left|\varepsilon_{1}\right| \mathbf{1}_{\left\{\left|\varepsilon_{1}\right| \geq h n^{1 / p_{\alpha}}\right\}} \leq c\left(h n^{1 / p_{\alpha}}\right)^{1-p}
$$

where $c=p(p-1)^{-1} \sup _{t>0} \mathbb{P}\left(\left|\varepsilon_{1}\right|>t\right)$. As

$$
\max _{1 \leq \ell \leq n} \ell^{-\alpha}\left|\sum_{j=k+1}^{k+\ell} \phi_{n}^{k+\ell-j} \mathbb{E}\left[\varepsilon_{j} \mathbf{1}_{\left\{\left|\varepsilon_{j}\right| \leq h n^{\left.1 / p_{\alpha}\right\}}\right.}\right]\right| \leq n^{1-\alpha} \mathbb{E}\left|\varepsilon_{1}\right| \mathbf{1}_{\left\{\left|\varepsilon_{j}\right| \geq h n^{1 / p_{\alpha}}\right\}}
$$

it follows that for every $h \geq h_{0}:=\max (1, c)$,

$n^{1-\alpha} \mathbb{E}\left|\varepsilon_{1}\right| \mathbf{1}_{\left\{\left|\varepsilon_{1}\right| \geq h n^{1 / p_{\alpha}}\right\}} \leq c h^{1-p} n^{1-\alpha+1 / p_{\alpha}-p / p_{\alpha}} \leq c h^{-1} n^{1-p / p_{\alpha}} n^{1 / p_{\alpha}} n^{-\alpha} \leq n^{1 / p_{\alpha}}$, recalling that $p \geq p_{\alpha}$ since $\alpha \leq \alpha_{p}$. Hence for every $n \geq 1$ and every $h \geq h_{0}$,

$$
\mathbb{P}\left(\max _{1 \leq \ell \leq n} \ell^{-\alpha}\left|\sum_{j=k+1}^{k+\ell} \phi_{n}^{k+\ell-j} \mathbb{E}\left[\varepsilon_{j} \mathbf{1}_{\left\{\left|\varepsilon_{j}\right| \leq h n^{\left.1 / p_{\alpha}\right\}}\right.}\right]\right|>h n^{1 / p_{\alpha}}\right)=0
$$


and we deduce

$$
P_{n, h}^{(1,2)} \leq \mathbb{P}\left(T_{n}^{(1,2,1)}>h n^{1 / p_{\alpha}}\right)
$$

with

$$
T_{n}^{(1,2,1)}:=\max _{1 \leq \ell<n} \ell^{-\alpha} \max _{1 \leq k \leq n-\ell}\left|\sum_{j=k+1}^{k+\ell} \phi_{n}^{k+\ell-j} \varepsilon_{j}^{\prime \prime}\right| .
$$

Since

$$
T_{n}^{(1,2,1)}=\max _{1 \leq \ell<n} \ell^{-\alpha} \max _{1 \leq k \leq n-\ell}\left|\sum_{j=1}^{\ell} \phi_{n}^{\ell-j} \varepsilon_{k+j}^{\prime \prime}\right| \leq \max _{1 \leq k \leq n} \max _{1 \leq \ell \leq n} \ell^{-\alpha}\left|\sum_{j=1}^{\ell} \phi_{n}^{\ell-j} \varepsilon_{k+j}^{\prime \prime}\right|
$$

we have

$$
\begin{aligned}
\mathbb{P}\left(T_{n}^{(1,2,1)} \geq h n^{1 / p_{\alpha}}\right) & \leq \sum_{k=1}^{n} \mathbb{P}\left(\max _{1 \leq \ell \leq n} \ell^{-\alpha}\left|\sum_{j=1}^{\ell} \phi_{n}^{\ell-j} \varepsilon_{k+j}^{\prime \prime}\right| \geq h n^{1 / p_{\alpha}}\right) \\
& =n \mathbb{P}\left(\max _{1 \leq \ell \leq n} \ell^{-\alpha}\left|\sum_{j=1}^{\ell} \phi_{n}^{\ell-j} \varepsilon_{j}^{\prime \prime}\right| \geq h n^{1 / p_{\alpha}}\right) .
\end{aligned}
$$

Let $K>1$ and let $M K=n$ where $M$ and $K$ (not necessarily integers) depend on $n$ in a way which will be precised later. Splitting the set

$$
\{1, \ldots, n\}=\bigcup_{m=1}^{M}(\mathbb{N} \cap((m-1) K, m K]),
$$

we have

$$
\begin{aligned}
\max _{1 \leq \ell \leq n} \ell^{-\alpha}\left|\sum_{j=1}^{\ell} \phi_{n}^{\ell-j} \varepsilon_{j}^{\prime \prime}\right| & \leq \max _{1 \leq m \leq M} \max _{(m-1) K<\ell \leq m K} \ell^{-\alpha}\left|\sum_{j=1}^{\ell} \phi_{n}^{\ell-j} \varepsilon_{j}^{\prime \prime}\right| \\
& \leq \max _{1 \leq m \leq M} \max _{(m-1) K<\ell \leq m K}[(m-1) K+1]^{-\alpha} \phi_{n}^{(m-1) K+1}\left|\sum_{j=1}^{\ell} \phi_{n}^{-j} \varepsilon_{j}^{\prime \prime}\right|
\end{aligned}
$$

This leads to

$$
\mathbb{P}\left(T_{n}^{(1,2,1)} \geq h n^{1 / p_{\alpha}}\right) \leq n \sum_{m=1}^{M} \mathbb{P}\left(\max _{(m-1) K<\ell \leq m K}\left|\sum_{j=1}^{\ell} \phi_{n}^{\ell-j} \varepsilon_{j}^{\prime \prime}\right| \geq h n^{1 / p_{\alpha}} \lambda_{m, K}\right)
$$

where $\lambda_{m, K}=[(m-1) K+1]^{\alpha} \phi_{n}^{-(m-1) K-1}$. Let $q>p \geq p_{\alpha}$, whose choice will be precised later. As $p_{\alpha} \leq p, \varepsilon_{1} \in \mathcal{L}_{p_{\alpha}}$, so by (36),

$$
\mathbb{E}\left|\varepsilon_{1}^{\prime \prime}\right|^{q} \leq \operatorname{ch}^{q-p_{\alpha}} n^{\left(q-p_{\alpha}\right) / p_{\alpha}},
$$


where the constant $c$ depends on $q, \alpha$ and the distribution of $\varepsilon_{1}$ only. Using successively Markov's, Doob's and Rosenthal's inequalities, we obtain

$$
\begin{aligned}
\mathbb{P}\left(T_{n}^{(1,2,1)} \geq h n^{1 / p_{\alpha}}\right) & \leq n \sum_{m=1}^{M}\left(h n^{1 / p_{\alpha}} a_{m, K}\right)^{-q} \mathbb{E}\left|\sum_{j=1}^{m K} \phi_{n}^{-j} \varepsilon_{j}^{\prime \prime}\right|^{q} \\
& \leq c_{q} n \sum_{m=1}^{M}\left(h n^{1 / p_{\alpha}} a_{m, K}\right)^{-q}\left[\left(\sum_{j=1}^{m K} \phi_{n}^{-2 j}\right)^{q / 2}+\sum_{j=1}^{m K} \phi_{n}^{-q j} \mathbb{E}\left|\varepsilon_{1}^{\prime \prime}\right|^{q}\right] \\
& \leq C_{q} n\left(h n^{1 / p_{\alpha}}\right)^{-q} K^{-q \alpha} \sum_{m=1}^{M} m^{-q \alpha} \phi_{n}^{-q K}\left[\frac{1}{\left(1-\phi_{n}^{2}\right)^{q / 2}}+\frac{\mathbb{E}\left|\varepsilon_{1}^{\prime \prime}\right|^{q}}{\left(1-\phi_{n}^{q}\right)}\right],
\end{aligned}
$$

using (51) and recalling the restriction $h \geq h_{0} \geq 1$, we continue by

$$
\begin{aligned}
& \leq C_{q}^{\prime} n^{1-q / p_{\alpha}} h^{-p_{\alpha}} K^{-q \alpha} \sum_{m=1}^{M} m^{-q \alpha} \phi_{n}^{-q K}\left[\frac{1}{\left(1-\phi_{n}^{2}\right)^{q / 2}}+\frac{n^{q / p_{\alpha}-1}}{\left(1-\phi_{n}^{q}\right)}\right] \\
& \leq C_{q, \alpha} n^{1-q / p_{\alpha}} h^{-p_{\alpha}} \phi_{n}^{-q K} K^{-q \alpha}\left[\frac{1}{\left(1-\phi_{n}^{2}\right)^{q / 2}}+\frac{n^{q / p_{\alpha}-1}}{\left(1-\phi_{n}^{q}\right)}\right],
\end{aligned}
$$

since $q>1 / \alpha$. Now choosing $K \sim\left(1-\phi_{n}\right)^{-1}$ and observing that $\phi_{n}^{-q K} \sim \mathrm{e}^{q}$, we finally have

$$
\begin{aligned}
P\left(T_{n}^{(1,2,1)} \geq h n^{1 / p_{\alpha}}\right) & \leq C_{q, \alpha}^{\prime} n^{1-q / p_{\alpha}} h^{-p_{\alpha}}\left(1-\phi_{n}\right)^{q \alpha}\left[\frac{1}{\left(1-\phi_{n}^{2}\right)^{q / 2}}+\frac{n^{q / p_{\alpha}-1}}{\left(1-\phi_{n}^{q}\right)}\right] \\
& \leq C_{q, \alpha}^{\prime} h^{-p_{\alpha}}\left[\frac{n}{\left(n\left(1-\phi_{n}\right)\right)^{q / p_{\alpha}}}+\left(1-\phi_{n}^{q}\right)^{q \alpha-1}\right] .
\end{aligned}
$$

Now, the hypothesis (13) enables us to choose $q$ in such a way that $n(n) 1-$ $\left.\left.\phi_{n}\right)\right)^{-q / p_{\alpha}}$ remains bounded, namely $q>\max \left(p, p_{\alpha} / \delta\right)$, so we obtain $T_{n}^{(1,2,1)}=$ $O_{P}\left(n^{1 / p_{\alpha}}\right)$.

Estimate for $T_{n}^{(2)}$. As already seen in the proof of inequality (11),

$$
\max _{1 \leq \ell<n} \ell^{-\alpha}\left(1-\phi_{n}^{\ell}\right)=O\left(\left(1-\phi_{n}\right)^{\alpha}\right)
$$

so it remains only to check that

$$
\max _{1 \leq k \leq n}\left|y_{n, k}\right|=O_{P}\left(n^{1 / p_{\alpha}}\left(1-\phi_{n}\right)^{-\alpha}\right) .
$$

But this is known from [9, Lemma 1], with $o_{P}$ instead of $O_{P}$.

Estimate for $T_{n}^{(3)}$. Finally for $T_{n}^{(3)}$, we have $\mathbb{E} y_{n}^{2}=\sum_{k=1}^{n} \phi_{n}^{2(n-k)} \sigma^{2} \leq n \sigma^{2}$. Hence, $y_{n, n}=O_{P}\left(n^{1 / 2}\right)$ and we obtain $T_{n}^{(3)}=n^{-\alpha} O_{P}\left(n^{1 / 2}\right)$. 


\section{B Proofs of consistency lemmas}

\section{B.1 Proof of Lemma 3.1}

Recalling (1), (20) and (21), we note that

$$
\begin{aligned}
\widehat{\phi}_{n}-\phi_{n} & =\frac{\sum_{k=1}^{n} y_{n, k-1}\left(y_{n, k}-\phi_{n} y_{n, k-1}\right)}{\sum_{k=1}^{n} y_{n, k-1}^{2}} \\
& =\frac{\sum_{k=1}^{n} y_{n, k-1}\left(\varepsilon_{k}+a_{n, k}\right)}{\sum_{k=1}^{n} y_{n, k-1}^{2}} \\
& =\frac{\sum_{k=1}^{n} z_{n, k-1} \varepsilon_{k}+\sum_{k=1}^{n} \tau_{n, k-1} \varepsilon_{k}+\sum_{k=1}^{n} y_{n, k-1} a_{n, k}}{\sum_{k=1}^{n} y_{n, k-1}^{2}} .
\end{aligned}
$$

To obtain an upper bound for $\left|\widehat{\phi}_{n}-\phi_{n}\right|$, we treat separately the three sums in the above numerator.

First, since $k^{*} \geq \lambda n$,

$$
\sum_{k=1}^{n} y_{n, k-1}^{2} \geq \sum_{k=1}^{k^{*}} y_{n, k-1}^{2}=\sum_{k=1}^{k^{*}} z_{n, k-1}^{2} \geq \sum_{k=1}^{[\lambda n]} z_{n, k-1}^{2},
$$

whence

$$
\frac{\left|\sum_{k=1}^{n} z_{n, k-1} \varepsilon_{k}\right|}{\sum_{k=1}^{n} y_{n, k-1}^{2}} \leq \frac{\left|\sum_{k=1}^{n} z_{n, k-1} \varepsilon_{k}\right|}{\sum_{k=1}^{[\lambda n]} z_{n, k-1}^{2}}=\left|\widetilde{\phi}_{n}-\phi_{n}\right| \frac{\sum_{k=1}^{n} z_{n, k-1}^{2}}{\sum_{k=1}^{[\lambda n]} z_{n, k-1}^{2}},
$$

where $\widetilde{\phi}_{n}=\left(\sum_{k=1}^{n} z_{n, k} z_{n, k-1}\right) /\left(\sum_{k=1}^{n} z_{n, k-1}^{2}\right)$ is the least squares estimator of $\phi_{n}$ associated to the process $\left(z_{n, k}\right)$. As already observed, when $\left(y_{n, k}\right)$ satisfies $H_{A},\left(z_{n, k}\right)$ satisfies $H_{0}$ and is then a nearly nonstationary $\operatorname{AR}(1)$ process. By [7, Th. 1],

$$
\widetilde{\phi}_{n}-\phi_{n}=O_{P}\left(n^{-1 / 2}\left(1-\phi_{n}\right)^{1 / 2}\right)
$$

Rewriting this estimate as $O_{P}\left(\left(1-\phi_{n}\right)\left(n\left(1-\phi_{n}\right)\right)^{-1 / 2}\right)$ and recalling that $n\left(1-\phi_{n}\right)$ tends to infinity, we get

$$
\widetilde{\phi}_{n}-\phi_{n}=o_{P}\left(1-\phi_{n}\right) \text {. }
$$

Moreover by [7, Lem. 2],

$$
\frac{1-\phi_{n}^{2}}{n} \sum_{k=1}^{n} z_{n, k-1}^{2} \underset{n \rightarrow \infty}{\stackrel{\mathrm{P}}{\longrightarrow}} \sigma^{2} .
$$


Recalling that $\gamma_{n}=n\left(1-\phi_{n}\right)$ is assumed to be non decreasing in $n$ or regularly varying, it is easily deduced from this weak law of large numbers that

$$
\frac{\sum_{k=1}^{n} z_{n, k-1}^{2}}{\sum_{k=1}^{[n \lambda]} z_{n, k-1}^{2}}=O_{P}(1)
$$

Going back to (55) with the estimates (56) and (58), we obtain

$$
\frac{\left|\sum_{k=1}^{n} z_{n, k-1} \varepsilon_{k}\right|}{\sum_{k=1}^{n} y_{n, k-1}^{2}}=o_{P}\left(1-\phi_{n}\right)
$$

For the second sum in the numerator in (53), a simple variance computation provides

$$
\sum_{k=1}^{n} \tau_{n, k-1} \varepsilon_{k}=O_{P}\left(\left(\sum_{k=1}^{n} \tau_{n, k-1}^{2}\right)^{1 / 2}\right)
$$

Then

$$
\sum_{k=1}^{n} \tau_{n, k-1}^{2}=\sum_{k=1}^{n}\left(\sum_{j=1}^{k-1} \phi_{n}^{k-1-j} a_{n, j}\right)^{2}=a_{n}^{2} \sum_{k=1}^{n}\left(\sum_{j=1}^{k-1} \phi_{n}^{k-1-j} \mathbf{1}_{\mathbb{I}_{n}^{*}}(j)\right)^{2}
$$

and

$$
\begin{aligned}
\sum_{k=1}^{n}\left(\sum_{j=1}^{k-1} \phi_{n}^{k-1-j} \mathbf{1}_{\mathbb{I}_{n}^{*}}(j)\right)^{2} & =\sum_{k=k^{*}+1}^{m^{*}}\left(\sum_{j=k^{*}+1}^{k-1} \phi_{n}^{k-1-j}\right)^{2}+\sum_{k=m^{*}+1}^{n}\left(\sum_{j=k^{*}+1}^{m^{*}} \phi_{n}^{k-1-j}\right)^{2} \\
& =\sum_{k=k^{*}+1}^{m^{*}}\left(\frac{1-\phi_{n}^{k-k^{*}-1}}{1-\phi_{n}}\right)^{2}+\sum_{k=m^{*}+1}^{n} \phi_{n}^{2\left(k-m^{*}-1\right)}\left(\sum_{i=0}^{\ell^{*}-1} \phi_{n}^{i}\right)^{2} \\
& \leq \frac{l^{*}}{\left(1-\phi_{n}\right)^{2}}+\frac{l^{* 2}}{1-\phi_{n}^{2}}
\end{aligned}
$$

Since $a_{n}^{2} \ell^{*}=o\left(n\left(1-\phi_{n}\right)\right)$, it follows that

$$
\sum_{k=1}^{n} \tau_{n, k-1}^{2}=o\left(n\left(1-\phi_{n}\right)-1\right)+o\left(n \ell^{*}\right)
$$

Accounting (60), this gives

$$
\sum_{k=1}^{n} \tau_{n, k-1} \varepsilon_{k}=o_{P}\left(n^{1 / 2}\left(1-\phi_{n}\right)^{-1 / 2}\right)+o_{P}\left(\sqrt{n \ell^{*}}\right) .
$$


Next, by (54) and (57) and recalling that $n\left(1-\phi_{n}\right)$ is non decreasing or regularly varying in $n$, we see that

$$
\frac{1}{\sum_{k=1}^{n} y_{n, k-1}^{2}}=O_{P}\left(\frac{1-\phi_{[n \lambda]}}{[n \lambda]}\right)=O_{P}\left(\frac{[n \lambda]\left(1-\phi_{[n \lambda]}\right)}{[n \lambda]^{2}}\right)=O_{P}\left(\frac{1-\phi_{n}}{n}\right) .
$$

Finally, combining (61) and (62), we obtain

$$
\begin{aligned}
\frac{\left|\sum_{k=1}^{n} \tau_{n, k-1} \varepsilon_{k}\right|}{\sum_{k=1}^{n} y_{n, k-1}^{2}} & =o_{P}\left(\sqrt{\frac{1-\phi_{n}}{n}}\right)+o_{P}\left(\left(1-\phi_{n}\right) \sqrt{\frac{\ell^{*}}{n}}\right) \\
& =o_{P}\left(\frac{1-\phi_{n}}{\sqrt{n\left(1-\phi_{n}\right)}}\right)+o_{P}\left(1-\phi_{n}\right)=o_{P}\left(1-\phi_{n}\right),
\end{aligned}
$$

since $\ell^{*}<n$ and $n\left(1-\phi_{n}\right)$ tends to infinity.

To deal with the contribution of $\sum_{k=1}^{n} y_{n, k-1} a_{n, k}$, we note first that

$$
\begin{aligned}
\frac{\left|\sum_{k=1}^{n} y_{n, k-1} a_{n, k}\right|}{\sum_{k=1}^{n} y_{n, k-1}^{2}} & \leq \frac{\left(\sum_{k=1}^{n} y_{n, k-1}^{2}\right)^{1 / 2}\left(\sum_{k=1}^{n} a_{n, k}^{2}\right)^{1 / 2}}{\sum_{k=1}^{n} y_{n, k-1}^{2}} \\
& =\frac{\sqrt{\ell^{*}}\left|a_{n}\right|}{\left(\sum_{k=1}^{n} y_{n, k-1}^{2}\right)^{1 / 2}} \\
& =O_{P}\left(\sqrt{\ell^{*}}\left|a_{n}\right| \sqrt{\frac{1-\phi_{n}}{n}}\right),
\end{aligned}
$$

using (62). Due to (24), this gives

$$
\frac{\left|\sum_{k=1}^{n} y_{n, k-1} a_{n, k}\right|}{\sum_{k=1}^{n} y_{n, k-1}^{2}}=o_{P}\left(1-\phi_{n}\right) .
$$

Going back to the decomposition (53) with the estimates (59), (63) and (64), we conclude that $\left|\widehat{\phi}_{n}-\phi_{n}\right|=o_{P}\left(1-\phi_{n}\right)$.

\section{B.2 Proof of Lemma 3.2}

We use

$$
\sum_{j=1}^{n} \tau_{n, k-1}=\frac{a_{n}}{1-\phi_{n}}\left(\ell^{*}-\phi_{n}^{n-m^{*}} \frac{1-\phi_{n}^{\ell^{*}}}{1-\phi_{n}}\right) .
$$


To prove (25) we have to consider all the possible configurations of the sets $\{k+1, \ldots, k+\ell\}$ and $\left\{k^{*}+1, \ldots, k^{*}+\ell^{*}\right\}$. There are six configurations $I_{1}, \ldots, I_{6}$. Denote for $v=1, \ldots, 6$

$$
T_{\alpha, n}^{(v)}=\max _{k, \ell \in I_{v}} \ell^{-\alpha}\left|\sum_{j=k+1}^{k+\ell} \tau_{n, j-1}-\frac{\ell}{n} \sum_{j=1}^{n} \tau_{n, j-1}\right| .
$$

First consider configuration $I_{1}:=\left\{k, \ell:\left[k^{*}+1, m^{*}\right] \subset[k+1, k+\ell]\right\}$. We easily obtain

$$
\begin{aligned}
\sum_{j=k+1}^{k+\ell} \tau_{n, j-1} & =a_{n}\left[\sum_{j=k^{*}+1}^{m^{*}} \sum_{i=0}^{j-k^{*}-2} \phi_{n}^{i}+\phi_{n}^{-1} \sum_{j=m^{*}+1}^{k+\ell} \phi_{n}^{j} \sum_{i=k^{*}+1}^{m^{*}} \phi_{n}^{-i}\right] \\
& =\frac{a_{n}}{1-\phi_{n}}\left[\ell^{*}-\phi_{n}^{k+\ell-m *} \frac{1-\phi_{n}^{\ell *}}{1-\phi_{n}}\right] .
\end{aligned}
$$

Together with (65) we find

$$
\begin{aligned}
T_{\alpha, n}^{(1)} & =\frac{\left|a_{n}\right|}{1-\phi_{n}} \max _{k, \ell \in I_{1}} \ell^{-\alpha}\left|\ell^{*}(1-\ell / n)-\frac{1-\phi_{n}^{\ell *}}{1-\phi_{n}}\left(\phi_{n}^{k+\ell-m *}-(\ell / n) \phi_{n}^{n-m^{*}}\right)\right| \\
& \leq \frac{3\left|a_{n}\right|}{1-\phi_{n}} \ell^{*(1-\alpha)} .
\end{aligned}
$$

Now let us turn to second configuration $I_{2}:=\{k, \ell:[k+1, k+\ell] \subset$ $\left.\left[k^{*}+1, m^{*}\right]\right\}$. Obviously

$$
\sum_{j=k+1}^{k+\ell} \tau_{n, j-1}=\frac{a_{n}}{1-\phi_{n}}\left(\ell-\frac{\phi_{n}^{k-k^{*}}\left(1-\phi_{n}^{\ell}\right)}{1-\phi_{n}}\right),
$$

so

$$
\begin{aligned}
T_{\alpha, n}^{(2)} & =\frac{\left|a_{n}\right|}{1-\phi_{n}} \max _{k, \ell \in I_{2}} \ell^{-\alpha}\left|\ell-\frac{\phi_{n}^{k-k^{*}}\left(1-\phi_{n}^{\ell}\right)}{1-\phi_{n}}-\frac{\ell}{n}\left(\ell^{*}-\phi_{n}^{n-m^{*}} \frac{1-\phi_{n}^{\ell^{*}}}{1-\phi_{n}}\right)\right| \\
& \leq \frac{4\left|a_{n}\right|}{1-\phi_{n}} \ell^{*(1-\alpha)} .
\end{aligned}
$$

If we consider the third configuration $I_{3}:=\left\{k, \ell: k+1<k^{*}+1 \leq k+\ell<\right.$ $\left.m^{*}\right\}$, we have

$$
\begin{aligned}
\sum_{j=k+1}^{k+\ell} \tau_{n, j-1} & =a_{n} \sum_{j=k+1}^{k+\ell} \sum_{i=1}^{j-1} \phi_{n}^{j-1-i} \mathbf{1}_{\mathbb{I}_{n}^{*}}(i)=a_{n} \sum_{j=k^{*}+1}^{k+\ell} \sum_{i=k^{*}+1}^{j-1} \phi_{n}^{j-1-i} \\
& =\frac{a_{n}}{1-\phi_{n}}\left(\left(k+\ell-k^{*}\right)-\frac{1-\phi_{n}^{k+\ell-k^{*}}}{1-\phi_{n}}\right) .
\end{aligned}
$$


Since $k+\ell-k^{*} \leq \ell^{*}$, then it is easy to see, that

$$
\begin{aligned}
T_{\alpha, n}^{(3)} & =\frac{\left|a_{n}\right|}{1-\phi_{n}} \max _{k, \ell \in I_{3}} \ell^{-\alpha}\left|\left(k+\ell-k^{*}\right)-\frac{1-\phi_{n}^{k+\ell-k^{*}}}{1-\phi_{n}}-\frac{\ell}{n}\left(\ell^{*}-\phi_{n}^{n-m^{*}} \frac{1-\phi_{n}^{\ell^{*}}}{1-\phi_{n}}\right)\right| \\
& \leq \frac{4\left|a_{n}\right|}{1-\phi_{n}} \ell^{*(1-\alpha)} .
\end{aligned}
$$

Next, fourth configuration is $I_{4}:=\left\{k, \ell: k^{*}+1<k+1 \leq m^{*}<k+\ell\right\}$. Now

$$
\sum_{j=k+1}^{k+\ell} \tau_{n, j-1}=\frac{a_{n}}{1-\phi_{n}}\left[\left(m^{*}-k\right)-\phi_{n}^{k-k^{*}} \frac{1-\phi_{n}^{m^{*}-k}}{1-\phi_{n}}+\left(1-\phi_{n}^{k+\ell-m^{*}}\right) \frac{1-\phi_{n}^{\ell^{*}}}{1-\phi_{n}}\right]
$$

together with (65) and $m^{*}-k \leq \ell^{*}$ gives the estimate

$$
\begin{aligned}
T_{\alpha, n}^{(4)}= & \frac{\left|a_{n}\right|}{1-\phi_{n}} \max _{k, \ell \in I_{4}} \ell^{-\alpha} \mid\left(m^{*}-k\right)-\phi_{n}^{k-k^{*}} \frac{1-\phi_{n}^{m^{*}-k}}{1-\phi_{n}}+\left(1-\phi_{n}^{k+\ell-m^{*}}\right) \frac{1-\phi_{n}^{\ell^{*}}}{1-\phi_{n}} \\
& -\frac{\ell}{n}\left(\ell^{*}-\phi_{n}^{n-m^{*}} \frac{1-\phi_{n}^{\ell^{*}}}{1-\phi_{n}}\right) \mid \leq \frac{5\left|a_{n}\right|}{1-\phi_{n}} \ell^{*(1-\alpha)} .
\end{aligned}
$$

From the fifth configuration $I_{5}:=\left\{k, \ell: m^{*}<k+1<k+\ell\right\}$, we get

$$
\sum_{j=k+1}^{k+\ell} \tau_{n, j-1}=\frac{a_{n}}{1-\phi_{n}} \cdot \phi_{n}^{k-m^{*}} \frac{\left(1-\phi_{n}^{\ell}\right)\left(1-\phi_{n}^{\ell^{*}}\right)}{1-\phi_{n}}
$$

and together with (65) the estimate is

$$
\begin{aligned}
T_{\alpha, n}^{(5)} & =\frac{\left|a_{n}\right|}{1-\phi_{n}} \max _{k, \ell \in I_{5}} \ell^{-\alpha}\left|\phi_{n}^{k-m^{*}} \frac{\left(1-\phi_{n}^{\ell}\right)\left(1-\phi_{n}^{\ell^{*}}\right)}{1-\phi_{n}}-\frac{\ell}{n}\left(\ell^{*}-\phi_{n}^{n-m^{*}} \frac{1-\phi_{n}^{\ell^{*}}}{1-\phi_{n}}\right)\right| \\
& \leq \frac{3\left|a_{n}\right|}{1-\phi_{n}} \ell^{*(1-\alpha)} .
\end{aligned}
$$

Finally sixth configuration $I_{6}:=\left\{k, \ell: k+1<k+\ell \leq k^{*}\right\}$ gives us

$$
\sum_{j=k+1}^{k+\ell} \tau_{n, j-1}=0
$$

Thus

$$
T_{\alpha, n}^{(6)}=\frac{\left|a_{n}\right|}{1-\phi_{n}}\left|\ell^{*}-\phi_{n}^{n-m^{*}} \frac{1-\phi_{n}^{\ell^{*}}}{1-\phi_{n}}\right| \max _{k, \ell \in I_{6}} \ell^{-\alpha} \frac{\ell}{n} \leq \frac{2\left|a_{n}\right|}{1-\phi_{n}} \ell^{*(1-\alpha)} .
$$

So collecting all the estimates of $T_{\alpha, n}^{(v)}, v=1, \ldots, 6$ we obtain (25). 


\section{References}

[1] Levin B, Kline J. The CUSUM test of homogeneity with an application in spontaneous abortion epidemiology. Statistics in Medicine. 1985; 4:469-488.

[2] Commenges D, Seal J, Pinatel F. Inference about a change point in experimental neurophysiology. Math Biosc. 1986;80:81-108.

[3] Avery P, Henderson A. Detecting a changed segment in DNA sequences. J Roy Statist Soc Ser C. 1999;48:489-503.

[4] Broemeling L, Tsurumi H. Econometrics and structural change. Marcel Dekker, New York; 1987.

[5] Račkauskas A, Suquet C. Hölder norm test statistics for epidemic change. Journal of statistical planning and inference. 2004;126(2):495520.

[6] Gombay E. Testing for change-points with rank and sign statistics. Statistics \& Probability Letters. 1994;20:49-55.

[7] Giraitis L, Phillips P. Uniform limit theory for stationary autoregression. Journal of time series analysis. 2006;27(1):51-60.

[8] Bingham N, Goldie C, Teugels J. Regular variation. encyclopedia of mathematics and its applications. Cambridge: Cambridge Univ. Press; 1987.

[9] Markevičiūtė J, Račkauskas A, Suquet C. Functional limit theorems for sums of nearly nonstationary processes. Lithuanian Mathematical Journal. 2012;52(3):282-296.

[10] Račkauskas A, Suquet C. Necessary and sufficient condition for the functional central limit theorem in Hölder spaces. Journal of Theoretical Probability. 2004;17(1):221-243.

[11] Mikosch T, Račkauskas A. The limit distribution of the maximum increment of random walk with regularly varying jump size distribution. Bernoulli. 2010;16(4):1016-1038.

[12] Fazekas I, Klesov O. A general approach to the strong laws of large numbers. Theory of Probab Appl. 2000;45(3):568-583.

[13] Embrechts P, Klüppelberg C, Mikosch T. Modelling extremal events for insurance and finance. Berlin: Springer; 1997. 